\title{
Pertuzumab in the treatment of HER2-positive breast cancer: an evidence-based review of its safety, efficacy, and place in therapy
}

This article was published in the following Dove Press journal: Core Evidence

Kei Ishii

Nao Morii

Hiroyasu Yamashiro

Department of Breast Surgery, Tenri Hospital, Tenri, Japan
Correspondence: Kei Ishii

Breast Surgery, Tenri Hospital, 200,

Mishima town, Tenri City, Nara

Prefecture 632-0015, Japan

Tel +8I 7436356 II

Email keiishii@tenriyorozu.jp

\begin{abstract}
Human epidermal growth factor receptor 2 (HER2)-positive breast cancer is a distinct subset of breast cancer that results from overexpression of HER2 protein. Pertuzumab-a recombinant humanized monoclonal antibody that binds to the extracellular dimerization domain II of HER2 - was recently approved for adjuvant therapy and neoadjuvant therapy of HER2positive early breast cancer. As pertuzumab and trastuzumab bind to different domains of the extracellular dimerization domain of HER2, a combination therapy of pertuzumab and trastuzumab is beneficial for the treatment of metastatic cancer, advanced local cancer, or early cancer by dual HER2 blockage. Many clinical trials have been performed using pertuzumab for breast cancer patients; these include the CLEOPATRA trial for palliative therapy, the APHINITY trial for adjuvant therapy, and the NeoSphere and the TRYPHAENA trials for neoadjuvant therapy. These trials revealed pertuzumab to be a safe and effective drug regardless of the patient age and hormone receptor status. Notably, pertuzumab use was associated with severe cardiac toxicity in some cases; however, the risk of pertuzumab-induced cardiac dysfunction was low. The most common adverse effect associated with pertuzumab-use was diarrhea, but most cases were not severe. Several different chemotherapeutic agents have been investigated to determine optimal chemotherapeutic combinations for dual HER2 blockage. Some exploratory analyses indicate that pertuzumab treatment offered little benefit to patients with node-negative and small primary tumors; pertuzumab treatment was also found not be cost-effective. Further research will reveal the appropriate usage of pertuzumab for treating a subset of eligible patients.
\end{abstract}

Keywords: pertuzumab, HER2, breast cancer, trastuzumab

\section{Core evidence clinical impact summary}

\begin{tabular}{|c|c|c|}
\hline Outcome measure & Evidence & Implications \\
\hline $\begin{array}{l}\text { Disease-oriented } \\
\text { evidence }\end{array}$ & $\begin{array}{l}\text { Clinical } \\
\text { trials }\end{array}$ & $\begin{array}{l}\text { Pertuzumab has been shown to be safe and effective in metastatic and } \\
\text { early HER2-positive breast cancer. }\end{array}$ \\
\hline $\begin{array}{l}\text { Patient-oriented } \\
\text { evidence }\end{array}$ & $\begin{array}{l}\text { Clinical } \\
\text { trials }\end{array}$ & $\begin{array}{l}\text { Pertuzumab has been demonstrated to be effective for progressive growth } \\
\text { against trastuzumab single-agent treatment for metastatic HER2-positive } \\
\text { breast cancer. On the other hand, pertuzumab does not appear to be } \\
\text { beneficial in patients with node-negative, small primary tumors in adjuvant } \\
\text { and neoadjuvant therapy for early HER2-positive breast cancer patients. }\end{array}$ \\
\hline Economic evidence & Articles & $\begin{array}{l}\text { Cost-effectiveness of pertuzumab is controversial and it is important } \\
\text { to establish efficient methods for selecting which patients it is most } \\
\text { suitable for, in order to improve the cost-effectiveness. }\end{array}$ \\
\hline
\end{tabular}




\section{Introduction}

Breast cancer is the most frequently diagnosed cancer and is the leading cause of cancer-related death among women, with an estimated 1.7 million cases and more than 500,000 deaths attributed to it worldwide. ${ }^{1}$ The human epidermal growth factor receptor 2 (HER2) is overexpressed in roughly $15-20 \%$ of the breast cancers diagnosed., ${ }^{2,3}$ Before the development of HER2-targeting agents, overexpression of HER2 was associated with aggressive tumor proliferation and metastatic activity. ${ }^{3,4}$ High HER2 expression is an indicator of poor prognosis in breast cancer patients. ${ }^{5}$ Further understanding of the molecular mechanisms underlying HER2-positive oncogenesis has led to the development of a series of HER2-targeting agents, which have revolutionized the standard of care for HER2-positive breast cancers. ${ }^{6}$

The HER family consists of epidermal growth factor receptor (EGFR, also known as HER1), HER2, HER3, and HER4. This receptor family transduces signals by homo- or hetero-dimerization. ${ }^{7,8}$ HER1, HER3, and HER4 need to be ligand-bound in order to undergo conformational changes and rapid dimerization, whereas HER2 has a constitutively activated dimerization unit. ${ }^{5}$ Of the ten different HER homoand hetero-dimer combinations, those containing HER2 endure for a long time and transmit strong signals, and are therefore associated with malignant growth. ${ }^{9}$ Moreover, HER2 overexpression promotes formation of HER2 heterodimers. HER2/HER3 dimerization has a particularly strong intracellular signal activity. ${ }^{10-12}$ In contrast, dimer combinations that do not include HER2 have a weak signal activity. In non-cancerous tissues, these dimer combinations aid healthy cell growth, but will not lead to tumorigenesis. ${ }^{9}$ In conclusion, the HER 2 receptor plays a crucial role in the network of cell signaling processes that control tumor development.

The clinicopathological importance of HER2 signaling pathways for treating HER2-positive metastatic breast cancers has become well recognized since a trastuzumabbased therapy was first described by Baselga et al. ${ }^{13}$ Trastuzumab inhibits downstream signaling not only by blocking HER2 homo-dimerization ${ }^{14}$ and ligand-stimulated HER2/HER3 hetero-dimerization, ${ }^{15}$ but it also inhibits the cleavage of the HER2 extracellular domain that prevents production of an active, truncated HER2 fragment. ${ }^{16,17}$ Moreover, trastuzumab induces antibodydependent cell-mediated toxicity. ${ }^{18}$ The prognosis of both early-stage $^{19,20}$ and metastatic ${ }^{21}$ HER2-positive breast cancer patients has drastically improved by trastuzumab.
However, therapeutic resistance to trastuzumab has become an increasingly important clinical issue. Approximately $15 \%$ of patients relapse after therapy, which indicates the presence of de novo or acquired resistance. ${ }^{17,22}$ The mechanisms underlying the development of resistance to trastuzumab are still under active investigation. $^{23,24}$ Recently, various resistance mechanisms have been considered, ${ }^{25,26}$ including, but not limited to: 1) escape from antibody-dependent cell mediated cytotoxicity, 2) crosstalk between estrogen receptor and HER2 pathways, 3) intrinsic alterations in HER2 protein, 4) expression levels of HER2 mRNA and protein, 5) aberrant activation of pathways downstream of HER family proteins (ie PI3K/Akt/mTOR pathway), 6) alterations in apoptosis and cell cycle control, and 7) expression of other HER family proteins in the cellular membrane and their interaction. Ghost et al showed that trastuzumab blocks HER2 homodimer-, but not heterodimer-induced Erk (Extracellular Signal-regulated Kinase) $1 / 2$ activity. ${ }^{14}$ This finding suggests that HER2 homodimer-activated signaling cascades are different from those activated by heterodimers of HER2 and other members of the HER family. Therefore, other strategies are needed for silencing these heterodimer-induced signaling pathways.

Pertuzumab is a recombinant, humanized, monoclonal antibody that binds to the extracellular dimerization domain II of HER2. Domain II is located on the opposite side of domain IV, where trastuzumab binds. ${ }^{27}$ Pertuzumab inhibits heterodimerization of HER2 with HER1, HER3, HER $4,{ }^{28-30}$ and IGF-1 $\mathrm{R}^{31}$ thereby inhibiting tumor cell growth downstream to ligand-dependent HER2 heterodimerization (Figure 1). ${ }^{14}$ Pertuzumab efficiently inhibits in vitro tumor cell growth by blocking ligand-mediated HER2/HER3 heterodimerization, whereas trastuzumab is more efficient at inhibiting cell growth in the absence of HER3 ligand. ${ }^{28,32}$ HER2 overexpression in human breast cancers is associated with activation of HER3, but not of EGFR, highlighting the importance of therapeutic strategies that disrupt the critical HER2/HER3 heterodimer in HER2-positive breast cancers. ${ }^{32}$ These findings suggest that a combination of trastuzumab and pertuzumab can be used for treating HER2-overexpressing tumors. ${ }^{29}$

Other studies show that inhibition of HER2 phosphorylation in HER2-positive breast cancer cells by tyrosine kinase inhibitors (TKIs) targets HER1 and HER2. This leads to subsequent feedback upregulation of activated HER3, thus, limiting the inhibitory effect of TKIs. ${ }^{33,34}$ This regulation is supported by data showing that blocking 

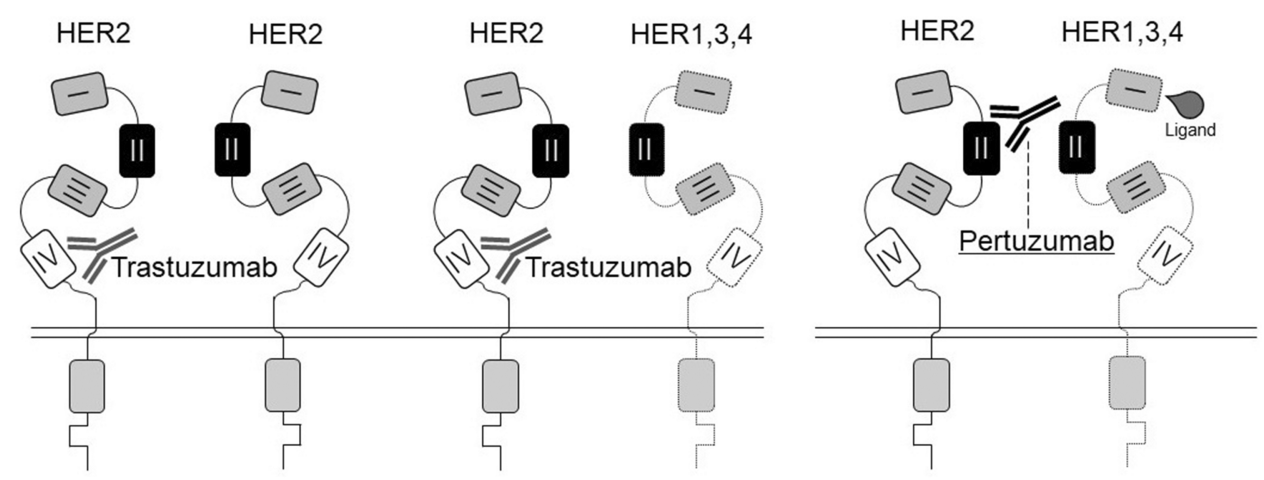

Figure I The scheme of the trastuzumab and pertuzumab action. Trastuzumab binds to the extracellular domain (ECD) IV of the HER2 receptor, preventing not only the spontaneous formation of homodimers (HER2-HER2) but also ligand-independent heterodimers (HER2-HER3, HER2-HERI, and HER2-HER4). Pertuzumab binds to the dimerization domain of the HER2 receptor (ECD II), preventing ligand-dependent HER2 heterodimerization. Adapted by permission from the American Association for Cancer Research: Metzger-Filho O, Winer EP, Krop I. Pertuzumab: Optimizing HER2 blockade. Clin Cancer Res. 2013;19(20):5552-5556. doi:I0.I I58/I078-0432.CCR-I3-05I 8. ${ }^{30}$

HER3 at the cell surface, using an anti-HER3 antibody, might be an effective approach to optimize antitumor action of the HER2 antagonist. However, pharmacologic inhibition of HER3 alone may not be an effective singleagent therapy for HER2-positive breast cancer. ${ }^{35}$

The importance of HER3-mediated signaling inhibition has also been demonstrated using xenograft models. Administration of pertuzumab to xenograft models already treated with trastuzumab induced tumor shrinkage, despite progressive growth against trastuzumab single-agent treatment. $^{27,36}$

Moreover, it has been shown that both trastuzumab and pertuzumab induce the activation of antibody-dependent cellular cytotoxicity $(\mathrm{ADCC})^{36}$ and complement-mediated cytotoxicity $(\mathrm{CDC})^{37}$ pathways, which are crucial aspects of immune system-mediated antitumor activity. Trastuzumab and pertuzumab independently activated ADCC against HER2-positive tumor cells (with equal potency). However, increase in ADCC efficiency was not observed when both agents were used together. Targeting the tumor cells with either trastuzumab or pertuzumab alone had only a minor effect on CDC. However, combination therapy using trastuzumab or pertuzumab was found to be effective at killing tumor cells only when the complement regulatory proteins (eg, CD46, CD55, and CD59) were neutralized to circumvent complement resistance. These preclinical studies reveal that combination therapy with pertuzumab and trastuzumab would be very helpful in combating HER2-positive breast cancer.

A single-arm, phase II study in 2010 (BO17929) revealed that the combination of pertuzumab and trastuzumab was active and well tolerated in patients with metastatic HER2-positive breast cancer whose tumor had progressed during prior trastuzumab therapy. ${ }^{38}$ After that, several clinical trials were performed. In this review, we focused on the following clinical trials: WO20698 trial $(\text { CLEOPATRA })^{39-41}$ in a palliative therapy, BO25126 trial (APHINITY) ${ }^{42}$ with adjuvant therapy, and WO20697 (NeoSphere) $)^{20,43}$ and BO22280 (TRYPHAENA) ${ }^{44,45}$ trials with neoadjuvant therapy. The outlines of the clinical trials are shown in Table 1.

\section{Safety \\ Cardiac dysfunction}

Among the potential side effects of HER2 targeted therapy, cardiac dysfunction is one that has potentially severe consequences. HER family members play a crucial role in normal cardiac development. ${ }^{46}$ Mice with embryonic lethal HER2 or HER4 knockout have malformed cardiac ventricles. ${ }^{47,48}$ Cardiac-restricted deletion of HER2 leads to dilated cardiomyopathy, and reduced tolerance to fluid overload in mice. ${ }^{49,50}$ It has been shown that heregulin stimulates proliferation and inhibits apoptosis in neonatal and adult cardiac myocytes that constitutively express HER2 and HER4. ${ }^{51}$ Thus, targeting HER2 may alter signals necessary for cardiac myocyte survival leading to cardiotoxicity. Trastuzumab-induced cardiotoxicity is the primary, dose-limiting adverse event in HER2-positive breast cancer patients treated with trastuzumab. ${ }^{52}$ In a retrospective review where the patients were administered with either a combination of trastuzumab, anthracycline, and cyclophosphamide, or a combination of trastuzumab and paclitaxel, the risks of the cardiotoxicity were $27 \%$ and $13 \%$, respectively. ${ }^{53}$ The rate of severe heart failure (New York Heart Association [NYHA] class III or IV) reportedly ranges from $0.5-4.1 \%$ in adjuvant therapy clinical trials 
Table I Summary of the CLEOPATRA, APHINITY, NeoSphere, and TRYPHAENA clinical trials

\begin{tabular}{|c|c|c|c|}
\hline Trial $^{\text {reference }}$ & Key Eligibility Criteria & Arms & $\begin{array}{l}\text { Primary } \\
\text { endpoint }\end{array}$ \\
\hline CLEOPATRA $^{39}$ & $\begin{array}{l}18 \text { years or older } \\
\text { metastatic } \\
\text { HER2+ }(\mathrm{IHC} 3+\text { or } \mathrm{FISH}+) \\
\text { ECOG PS }=0 \text { or I } \\
\text { LVEF }>50 \% \\
\text { no treatment for metastatic disease } \\
\text { no central nervous system metastasis }\end{array}$ & $\begin{array}{l}\text { Per+Tra+DTx } \\
\text { Placebo+Tra+DTx }\end{array}$ & PFS \\
\hline APHINITY 42 & $\begin{array}{l}18 \text { years or older } \\
\text { non metastatic, first time breast cancer } \\
\text { HER } 2+(\mathrm{IHC} 3+\text { or } \mathrm{FISH}+) \\
\text { ECOG PS }=0 \text { or } 1 \\
\text { tumor diameter }>1.0 \mathrm{~cm} \text { or node positive } \\
\text { LVEF }>55 \%\end{array}$ & $\begin{array}{l}\text { Per+Tra+Chemotherapy } \\
\text { Placebo+Tra+Chemotherapy } \\
\text { (Chemotherapy included anthracyclin } \\
\text { and/or taxane-base treatment) }\end{array}$ & IDFS \\
\hline NeoSphere $^{20}$ & $\begin{array}{l}\text { Non metastatic, unilateral breast cancer } \\
\text { no other malignancies } \\
\text { HER2 }+(\mathrm{IHC} 3+\text { or } \mathrm{FISH}+) \\
\text { tumor diameter }>2.0 \mathrm{~cm} \text { or inflammatory } \\
\text { LVEF }>55 \%\end{array}$ & $\begin{array}{l}\text { Tra+DTx followed by operation, FEC with Tra } \\
\text { Per+Tra+DTx followed by operation, FEC with Tra } \\
\text { Per+Tra followed by operation, FEC, and DTx with Tra } \\
\text { Per+DTx followed by operation, FEC with Tra }\end{array}$ & bpCR \\
\hline TRYPHAENA $^{44}$ & $\begin{array}{l}\text { Non metastatic, unilateral breast cancer } \\
\text { no exposure for anticancer therapy } \\
\text { HER2+ }(\mathrm{IHC} 3+\text { or } \mathrm{FISH}+\text { ) } \\
\text { tumor diameter }>2.0 \mathrm{~cm} \text { or inflammatory } \\
\text { LVEF }>55 \%\end{array}$ & $\begin{array}{l}\text { FEC+Per+Tra followed by DTx+Per+Tra, operation, and Tra } \\
\text { FEC followed by DTx+Per+Tra operation, and Tra } \\
\text { DTx+CBDCA+Per+Tra followed by operation and Tra }\end{array}$ & Incidence of LVSD \\
\hline
\end{tabular}

Notes: Key eligibility criteria, trial arms (regimens), and primary outcome of mainly evidential clinical trials for pertuzumab.

Abbreviations: HER2, Human epithelial growth factor receptor 2; IHC, immunohistochemistry; FISH, fluorescence in situ hybridization; PS, performance status; LVEF, left ventricular ejection fraction; Per, pertuzumab; Tra, trastuzumab; DTx, docetaxel; FEC, fluorouracil, epirubicin, and cyclophosphamide regimen; CBDCA, carboplatin; PFS, progression-free survival; IDFS, invasive disease-free survival; bPCR, breast pathological complete response; LVSD, left ventricular systolic dysfunction.

with trastuzumab. ${ }^{19,54-57}$ Therefore, cardiotoxicity was studied by researchers in each of the clinical trials. In the CLEOPATRA trial, ${ }^{39}$ left ventricular systolic dysfunction (any grade) was reported more frequently in the control group $(8.3 \%)$ than in the pertuzumab group $(4.4 \%)$. However, there was no significant difference in the percentage of severe left ventricular dysfunction in the control group versus the pertuzumab group. In the APHINITY trial, $^{42}$ primary cardiac events occurred in 17 patients $(0.7 \%)$ in the pertuzumab group, and in 8 patients $(0.3 \%)$ in the placebo group. Fifteen patients in the pertuzumab group, and 6 patients in the placebo group had NYHA class III or IV heart failure with a substantial decrease in left ventricular ejection fraction (LVEF); it is noteworthy that two patients in each group died from cardiac failure. In the pertuzumab group, a primary cardiac event occurred in 15 patients $(0.8 \%)$ in the anthracycline cohort, and 2 patients $(0.4 \%)$ in the non-anthracycline cohort. Secondary cardiac events occurred in 64 patients $(2.7 \%)$ in the pertuzumab group and in 67 patients $(2.8 \%)$ in the placebo group. In the NeoSphere trial, ${ }^{20}$ the mean maximum decrease in LVEF measurement was low (4-5\%), and was similar across treatment groups. No significant change was detected when a combination therapy of pertuzumab and trastuzumab was used; the LVEF did not decrease to less than $40 \%$ at any time during the study period in any of the patients. In the TRYPHAENA trial, ${ }^{44}$ the incidence of symptomatic left ventricular systolic dysfunction (LVSD), as well as significant reduction in LVEF, was low across all arms of the study. Two patients $(2.7 \%)$ in the pertuzumab treatment arm of the trial experienced symptomatic LVSD during neoadjuvant treatment. Only 1 of the 223 patients $(0.4 \%)$ who had received trastuzumab and pertuzumab in combination with standard chemotherapy drugs - fluorouracil, epirubicin, and cyclophosphamide (FEC)-developed symptomatic LVSD during the neoadjuvant treatment.

Similarly, in a phase II, open-label, multicenter, multinational cardiac safety study (BERENICE), ${ }^{58}$ the overall 
incidences of NYHA class III or IV heart failure during neoadjuvant treatment were low and consistent with those observed in the TRYPHAENA study. Notably, the BERENICE study involved a longer neoadjuvant period (8 versus 6 chemotherapy cycles). A retrospective study concluded that a combination of trastuzumab and pertuzumab did not increase the risk of cardiotoxicity following a doxorubicin-based regimen. $^{59}$ In the PERUSE trial $^{60}$ a global, open-label, single-arm, phase IIIb study evaluating the safety and tolerability of pertuzumab in combination with trastuzumab and a taxane-LVEF remained above $50 \%$ in $86 \%$ of the patients. As stated above, cardiotoxicity from pertuzumab treatment is significantly lower than that in trastuzumab treatment, and the combination of these agents with trastuzumab did not significantly increase the adverse cardiac events.

In these studies, cardiac dysfunction was resolved after the study was discontinued, and patients were given medication to remedy the symptoms. In in vitro studies, ranolazine, a drug used in the clinic to treat chronic angina and ischemia, attenuated not only the cardiotoxic side effects of trastuzumab, but also those of pertuzumab when used in combinatorial treatments in cardiac cell lines. ${ }^{61}$ These agents may rescue HER2-positive breast cancer patients from treatment-induced cardiac dysfunction. To prevent severe iatrogenic heart failure, scheduled monitoring of heart function is recommended.

\section{Other adverse effects}

Adverse effects from clinical trials are reported in Table 2. In the CLEOPATRA trial, ${ }^{39}$ the incidences of grade 3 or higher febrile neutropenia, and diarrhea were higher by at least $2 \%$ in the pertuzumab group as compared to the control group. Interestingly, the incidence of febrile neutropenia (grade 3 or higher) among patients from Asia was $12 \%$ in the control group, and $26 \%$ in the pertuzumab group. In all other geographic regions, the incidence was $10 \%$ or less in both groups. In the APHINITY trial, ${ }^{42}$ the largest absolute difference in adverse effects between the treatment groups was found for diarrhea $(9.8 \%$ in the pertuzumab group and $3.7 \%$ in the placebo group). During targeted therapy alone, after cessation of chemotherapy, the incidence of grade 3 or higher diarrhea was $0.5 \%$ in the pertuzumab group, and $0.2 \%$ in the placebo group. In the NeoSphere trial, ${ }^{20}$ the most frequently occurring adverse events were alopecia, neutropenia, diarrhea, nausea, fatigue, rash, and mucosal inflammation, but most adverse events were of grades 1 or 2 . The number of serious adverse events was similar in chemotherapy treatment arms, with 15-
20 serious adverse events per group in $10-17 \%$ of patients, but lower in the pertuzumab and trastuzumab treatment arm. In the TRYPHAENA trial, ${ }^{44}$ diarrhea, alopecia, and nausea (all grades) were reported in more than $50 \%$ of patients across all arms during the neoadjuvant treatment period. Neutropenia, febrile neutropenia, and leukopenia were the most frequently reported at grade 3 or higher. The most common severe adverse effect was febrile neutropenia.

\section{Diarrhea}

In these four trials, diarrhea is considered the most common adverse effect of pertuzumab. A meta-analysis showed that among all the adverse effects, the incidence of diarrhea (odds ratio $[\mathrm{OR}] 2.310,95 \%$ confidence interval [CI] 1.818-2.936), rash (OR 1.848, 95\% CI 1.094-3.122), and febrile neutropenia (OR 1.672, 95\% CI 1.130-2.474) were of statistical significance, which meant that pertuzumab played a prominent role in causing diarrhea. ${ }^{63}$ HER1 and HER2 are expressed on intestinal epithelial cell membranes, and act in concert to negatively regulate chloride secretion via the phosphatidylinositol 3-kinase (PI3K) and protein kinase $\mathrm{C}$ (PKC) pathways. ${ }^{64}$ As such, excess chloride secretion potentially contributes to secretory diarrhea observed in up to $87 \%$ of the patients treated with TKIs. ${ }^{64,65}$ This mechanism differs from that observed in chemotherapy-induced diarrhea, which is secondary to mucositis of the gastrointestinal tract. ${ }^{66}$ According to an article which summarizes data from the CLEOPATRA, NeoSphere, and TRYPHAENA trials, ${ }^{67}$ most diarrheal episodes by pertuzumab-containing regimens were grade 1 (range: $21-54 \%$ ) and grade 2 (range: $8-37 \%$ ). Moreover, among patients with metastatic breast cancer, $2 \%$ of the patients in the pertuzumab arm of the CLEOPATRA trial discontinued their participation because of diarrhearelated complications, compared to $0.5 \%$ of the participants in the control arm. None of the patients discontinued their participation after diarrheal episodes in the early-stage breast cancer studies (NeoSphere and TRYPHAENA). Irrespective of treatment, patients under 65 years old exhibited similar rates of all grades of diarrhea. However, in the pertuzumabcontaining arm, grade 3 or higher diarrhea occurred more frequently among patients older than 65 years of age. Elderly patients receiving pertuzumab also had higher rates of drug discontinuation, and dose delays from diarrhea compared to patients in the control arm. Asian patients appeared to have a higher incidence of all grades of diarrhea compared to white patients, who had grade 3 diarrhea or higher, regardless of treatment. However, dose delays due to diarrhea were more frequent in white patients $(8 \%)$ than in Asian patients $(4 \%)$. 
Table 2 Primary adverse effects reported from the CLEOPATRA, APHINITY, NeoSphere, and TRYPHAENA trial

\begin{tabular}{|c|c|c|c|c|c|c|c|c|}
\hline Trial & \multicolumn{4}{|c|}{ CLEOPATRA } & \multicolumn{4}{|c|}{ APHINITY } \\
\hline \multirow[t]{2}{*}{ Regimens } & \multicolumn{4}{|c|}{ Tra + DTx + } & \multicolumn{4}{|c|}{ Adjuvant chemotherapy with Tra + } \\
\hline & \multicolumn{2}{|c|}{ Placebo $(\mathrm{N}=397)$} & \multicolumn{2}{|c|}{$\operatorname{Per}(\mathrm{N}=407)$} & \multicolumn{2}{|c|}{ Placebo $(\mathrm{N}=\mathbf{2 4 0 5})$} & \multicolumn{2}{|c|}{$\operatorname{Per}(N=2364)$} \\
\hline Grade & Grade $1 / 2$ & Grade3/4 & Grade $1 / 2$ & Grade 3/4 & All grade & Grade $3 / 4 / 5$ & All grade & Grade $3 / 4 / 5$ \\
\hline Diarrhea & $46 \%$ & $5 \%$ & $67 \%$ & $8 \%$ & $45 \%$ & $4 \%$ & $71 \%$ & $10 \%$ \\
\hline Constipation & $25 \%$ & $1 \%$ & $15 \%$ & $0 \%$ & $32 \%$ & $0.3 \%$ & $29 \%$ & $0.5 \%$ \\
\hline Nausea & $42 \%$ & $0.5 \%$ & $42 \%$ & $1 \%$ & $65 \%$ & $2 \%$ & $69 \%$ & $2 \%$ \\
\hline Vomiting & $24 \%$ & $2 \%$ & $24 \%$ & $1 \%$ & $30 \%$ & $2 \%$ & $32 \%$ & $2 \%$ \\
\hline Decreased appetite & $3 \%$ & $0.5 \%$ & $12 \%$ & $0 \%$ & $20 \%$ & $0.4 \%$ & $24 \%$ & $0.8 \%$ \\
\hline Neutropenia & $50 \%$ & $46 \%$ & $53 \%$ & $49 \%$ & $23 \%$ & $10 \%$ & $25 \%$ & $16 \%$ \\
\hline Febrile Neutropenia & $8 \%$ & $7 \%$ & $14 \%$ & $13 \%$ & $11 \%$ & $11 \%$ & $12 \%$ & $12 \%$ \\
\hline Fatigue & $37 \%$ & $3 \%$ & $37 \%$ & $2 \%$ & $44 \%$ & $3 \%$ & $49 \%$ & $4 \%$ \\
\hline Asthenia & $30 \%$ & $2 \%$ & $26 \%$ & $2 \%$ & $21 \%$ & $2 \%$ & $21 \%$ & $1 \%$ \\
\hline Rash & $24 \%$ & $0.8 \%$ & $34 \%$ & $0.7 \%$ & $20 \%$ & $0.2 \%$ & $26 \%$ & $0.4 \%$ \\
\hline Peripheral edema & $30 \%$ & $0.8 \%$ & $23 \%$ & $0.5 \%$ & $20 \%$ & $0.2 \%$ & $17 \%$ & 0 \\
\hline Mucosal inflammation & $20 \%$ & $1 \%$ & $28 \%$ & $1 \%$ & $19 \%$ & $0.7 \%$ & $23 \%$ & $2 \%$ \\
\hline Alopecia & $60 \%$ & $0.3 \%$ & $61 \%$ & $0 \%$ & $67 \%$ & $<0.1 \%$ & $67 \%$ & $<0.1 \%$ \\
\hline Trial & \multicolumn{8}{|c|}{ NeoSphere (Neoadjuvant period) } \\
\hline Regimens & \multicolumn{2}{|l|}{ Tra + DTx } & \multicolumn{2}{|c|}{ Per + Tra +DTx } & \multicolumn{2}{|l|}{ Per +Tra } & \multicolumn{2}{|l|}{ Per + DTx } \\
\hline & \multicolumn{2}{|l|}{$(N=107)$} & \multicolumn{2}{|l|}{$(N=107)$} & \multicolumn{2}{|l|}{$(N=108)$} & \multicolumn{2}{|l|}{$(\mathrm{N}=94)$} \\
\hline Grade & All grade & Grade $3 / 4 / 5$ & All grade & Grade $3 / 4 / 5$ & All grade & Grade $3 / 4 / 5$ & All grade & Grade $3 / 4 / 5$ \\
\hline Diarrhea & $34 \%$ & $4 \%$ & $46 \%$ & $6 \%$ & $28 \%$ & 0 & $54 \%$ & $4 \%$ \\
\hline Constipation & No data & No data & No data & No data & No data & No data & No data & No data \\
\hline Nausea & $36 \%$ & 0 & $38 \%$ & 0 & $14 \%$ & 0 & $36 \%$ & $1 \%$ \\
\hline Vomiting & $12 \%$ & 0 & $13 \%$ & 0 & $5 \%$ & 0 & $16 \%$ & $2 \%$ \\
\hline Decreased appetite & $7 \%$ & 0 & $14 \%$ & 0 & $2 \%$ & 0 & $15 \%$ & 0 \\
\hline Neutropenia & $64 \%$ & $59.0 \%$ & $50 \%$ & $45 \%$ & $0.9 \%$ & $0.9 \%$ & $65 \%)$ & $57 \%$ \\
\hline Febrile Neutropenia & $7 \%$ & $7 \%$ & $8 \%$ & $8 \%$ & 0 & 0 & $7 \%$ & $7 \%$ \\
\hline Fatigue & $27 \%$ & 0 & $26 \%$ & $0.9 \%$ & $12 \%$ & 0 & $26 \%$ & $1 \%$ \\
\hline Asthenia & $18 \%$ & 0 & $21 \%$ & $2 \%$ & $3 \%$ & 0 & $16.0 \%$ & $2 \%$ \\
\hline Rash & $21 \%$ & $2 \%$ & $26 \%$ & $2 \%$ & $11 \%$ & 0 & $29 \%$ & $1 \%$ \\
\hline Peripheral edema & $10 \%$ & 0 & $3 \%$ & 0 & $0.9 \%$ & 0 & $5 \%$ & 0 \\
\hline Mucosal inflammation & $21 \%$ & 0 & $26 \%$ & $2 \%$ & $3 \%$ & 0 & $26 \%$ & 0 \\
\hline Alopecia & $66 \%$ & 0 & $65 \%$ & 0 & $3 \%$ & 0 & $67 \%$ & 0 \\
\hline
\end{tabular}

(Continued) 
Table 2 (Continued).

\begin{tabular}{|c|c|c|c|c|c|c|}
\hline \multirow{3}{*}{$\begin{array}{l}\text { Trial } \\
\text { Regimens }\end{array}$} & \multicolumn{6}{|c|}{ TRYPHAENA (Neoadjuvant period) } \\
\hline & \multicolumn{2}{|c|}{$\begin{array}{l}\text { FEC + Per + Her followed by Per + Tra + } \\
\text { DTx }\end{array}$} & \multicolumn{2}{|c|}{$\begin{array}{l}\text { FEC Followed by Per }+ \text { Tra }+ \\
\text { DTx }\end{array}$} & \multicolumn{2}{|c|}{ Per + Her + DTx + CDBCA } \\
\hline & \multicolumn{2}{|l|}{$(N=72)$} & \multicolumn{2}{|l|}{$(N=75)$} & \multicolumn{2}{|l|}{$(N=76)$} \\
\hline Grade & All grade & Grade $3 / 4 / 5$ & All grade & Grade $3 / 4 / 5$ & All grade & Grade $3 / 4 / 5$ \\
\hline Diarrhea & $61 \%$ & $4 \%$ & $61 \%$ & $5 \%$ & $72 \%$ & $12 \%$ \\
\hline Constipation & $18 \%$ & 0 & $23 \%$ & 0 & $16 \%$ & 0 \\
\hline Nausea & $53 \%$ & 0 & $53 \%$ & $3 \%$ & $45 \%$ & 0 \\
\hline Vomiting & $40 \%$ & 0 & $36 \%$ & $3 \%$ & $39 \%$ & $5 \%$ \\
\hline Decreased appetite & $21 \%$ & 0 & $11 \%$ & 0 & $21 \%$ & 0 \\
\hline Neutropenia & $51 \%$ & $47 \%$ & $47 \%$ & $43 \%$ & $49 \%$ & $46 \%$ \\
\hline Febrile Neutropenia & $18 \%$ & $18 \%$ & $9 \%$ & $9 \%$ & $17 \%$ & $17 \%$ \\
\hline Fatigue & $36 \%$ & 0 & $36 \%$ & 0 & $42 \%$ & $4 \%$ \\
\hline Asthenia & $10 \%$ & 0 & $15 \%$ & $1 \%$ & $13 \%$ & $1 \%$ \\
\hline Rash & $19 \%$ & 0 & $11 \%$ & 0 & $21 \%$ & $1 \%$ \\
\hline Peripheral edema & $11 \%$ & 0 & $4 \%$ & 0 & $9 \%$ & 0 \\
\hline Mucosal inflammation & $24 \%$ & 0 & $20 \%$ & 0 & $17 \%$ & $1 \%$ \\
\hline Alopecia & $49 \%$ & 0 & $52 \%$ & 0 & $55 \%$ & 0 \\
\hline
\end{tabular}

Notes: The main adverse effects observed in each trial. Main data were from respective articles, ${ }^{20,39,42,44}$ and some detailed data were obtained from prescription information with regard to Perjeta ${ }^{\circledR}$. Available from: https://www.gene.com/download/pdf/perjeta_prescribing.pdf. ${ }^{62}$

Abbreviations: Per, pertuzumab; Tra, trastuzumab; $\overline{D T x}$, docetaxel; FEC, fluorouracil; epirubicin, and cyclophosphamide regimen; CBDCA, carboplatin.

A study of elderly women in South Asia showed no difference between the rate of diarrheal episodes in pertuzumabcontaining regimens (55\%), and non-pertuzumab-containing regimens (54\%) in neoadjuvant therapy. ${ }^{68}$ These results suggest that diarrhea is important in Asian or elderly patients populations.

Among patients with diarrhea, a larger proportion of patients across all pertuzumab-containing arms in the CLEOPATRA, NeoSphere, and TRYPHAENA trials received antidiarrheal treatment relative to the study arms not containing pertuzumab. Loperamide was the most frequently prescribed medication, and was administrated to $31-60 \%$ of patients who experienced diarrhea. ${ }^{67}$ Loperamide is a phenylpiperidine opioid that possesses antisecretory properties; it slows intestinal transit time by stimulating $\mu$-opioid receptors in the myenteric plexus, and blocks the intestinal calcium channels. ${ }^{69}$ The efficacy of loperamide in treating pertuzumab-associated diarrhea is unknown. Crofelemer is currently in a phase II, randomized, open-label trial to prevent and prophylactically treat pertuzumab-induced therapeutic diarrhea (HALT-D).$^{70}$ Further investigation is warranted.

\section{Efficacy}

\section{Palliative therapy}

In a single arm, phase II study (BO17929 trial), treatment with pertuzumab and trastuzumab was beneficial in patients with HER2-positive metastatic breast cancer. ${ }^{38}$ In this study, 66 patients with advanced HER2-positive breast cancer, where disease progression had occurred prior to trastuzumab-based therapy, received trastuzumab weekly or every three weeks, and pertuzumab every three weeks (840 mg loading dose, then $420 \mathrm{mg}$ every three weeks). The objective response rate was $24.2 \%$, and the clinical benefit rate was $50 \%$.

In the CLEOPATRA trial, the median, independently assessed progression-free survival (PFS) was prolonged from 12.4 months in the control group, to 18.5 months in the pertuzumab group (hazard ratio [HR] for progression or death, $0.62 ; 95 \%$ CI, $0.51-0.75 ; P<0.001)$. Pertuzumab decreased the HR regardless of whether trastuzumab had been prescribed with or without adjuvant or neoadjuvant chemotherapy. In the final analysis (median follow-up 
period was about 50 months for each arm), ${ }^{41} 168$ deaths were reported among 402 patients $(41.8 \%)$ in the pertuzumab group, and 221 deaths were reported among 406 patients $(54.4 \%)$ in the control group (HR favoring the pertuzumab group, $0.68 ; 95 \% \mathrm{CI}, 0.56-0.84 ; P<0.001)$. The median overall survival (OS) was 56.5 months $(95 \%$ CI, 49.3 to not reached) in the pertuzumab group, and 40.8 months (95\% CI, 35.8-48.3) in the control group, a difference of 15.7 months. The median duration of response was 20.2 months in the pertuzumab group (95\% CI, 16.0-24.0), and 12.5 months in the control group (95\% CI, 10.0-15.0). The overall response rate was $80.2 \%$. From this trial, first-line therapy with pertuzumab, trastuzumab, and docetaxel significantly improved OS among patients with HER2-positive metastatic breast cancer, compared with placebo, trastuzumab, and docetaxel.

\section{Adjuvant therapy}

In the APHINITY trial, ${ }^{42}$ invasive disease events were reported in 171 patients $(7.1 \%)$ in the pertuzumab group, and 210 patients $(8.7 \%)$ in the placebo group. The rate of survival from lack of invasive disease over 3 years was $94.1 \%$ in the pertuzumab group, and $93.2 \%$ in the placebo group, with a HR for an invasive disease event of 0.81 (95\% CI, 0.66-1.00; $P=0.045)$ in favor of pertuzumab. If the occurrence of a second primary non-breast cancer was included, the number of patients with an event increased to 189 in the pertuzumab group, and 230 in the placebo group, resulting in a significant difference between the groups (HR, $0.82 ; 95 \% \mathrm{CI}, 0.68$ to $0.99 ; P=0.04$ ). The addition of pertuzumab to chemotherapy and trastuzumab as an adjuvant treatment also improved outcomes among patients with HER2-positive early breast cancer.

\section{Neoadjuvant therapy}

In the NeoSphere trial, ${ }^{20}$ the primary endpoint was pathological complete response (pCR) rate in the breast (defined as ypT0/by TNM classification of malignant tumors). A pCR was achieved in 31 of the 107 women administered with trastuzumab plus docetaxel $(29.0 \%, 95 \%$ CI $20.6-$ $38.5 \%)$, compared to 49 in women $(n=107)$ administered with a combination of pertuzumab, trastuzumab, and docetaxel $(45.8 \%$, CI $36.1-55.7 \%)(P=0.0141)$. In comparison, 23 of the 96 women administered with pertuzumab plus docetaxel had pCR $(24.0 \%, 15.8-33.7 \%)$, and 18 of the 107 women treated with both anti-HER2 antibodies but without chemotherapy (16.8\%, CI $10.3-25.3 \%)$ had pCR.
Most patients achieved an objective response (complete response or partial response) in the primary lesion. As noted for $\mathrm{pCR}$, the greatest clinical response was reported in pertuzumab, trastuzumab, and docetaxel study arms. The investigators noted that there were not many patients with insufficient therapeutic responses during the neoadjuvant treatment period, although numbers were higher in the study arm with anti-HER2 antibodies without chemotherapy. There were no patients in the trastuzumab and docetaxel arm, 1 patient in pertuzumab, trastuzumab, and docetaxel arm, 7 patients in the pertuzumab and trastuzumab arm, and 1 patient in pertuzumab and docetaxel arm. In the TRYPHAENA trial, ${ }^{44}$ the primary endpoint was safety and $\mathrm{pCR}$ rate in the breast. In this trial, all the patients were prescribed pertuzumab with chemotherapy. pCR rate in the breast was consistently high and similar across all treatment groups (approximately 60\%). This result was similar to that of the NeoSphere trial. When pCR was defined as ypT0 and ypN0, about $50 \%$ of patients achieved $\mathrm{pCR}$.

The NeoSphere study was not designed to assess long term outcomes, but a 5-year PFS rate was calculated to be $86 \%$ for the pertuzumab, trastuzumab, and docetaxel study arm (95\% CI: $77-91 \%$ ), compared to $81 \%$ for the trastuzumab and docetaxel study arm (95\% CI: $71-87 \%)$. The HR for PFS was 0.69 (95\% CI: $0.34-1.40)$. The 5-year disease-free survival (DFS) rates were $81 \%$ in the trastuzumab and docetaxel study arm, and $84 \%$ in the pertuzumab, trastuzumab, and docetaxel study arm. The HR of DFS was 0.60 (95\% CI: 0.28-1.27). ${ }^{71}$

The overall response rate for locally recurrent or metastatic HER2-positive breast cancer was $80 \%$ in the CLEOPATRA and the PERUSE trials, ${ }^{60}$ The pCR rate was approximately $60 \%$ in the BERENICE trial, ${ }^{58} 67-$ $68 \%$ in the TRAIN-2 trial $^{72}$ an open-label, randomized controlled, phase III trial-and about $50 \%$ in the KRISTINE trial, ${ }^{73}$ a randomized, multicenter, open-label, phase III trial. These results suggested that pertuzumab can be effectively used in combination with trastuzumab and chemotherapy for treating HER2-positive breast cancer patients.

\section{Place in therapy \\ Clinical features of patients}

Most of the clinical trials revealed efficacy of pertuzumab in treating HER2-positive early, advanced, and metastatic breast cancer. However, subgroup analysis from each trial 
pointed out that some patient populations did not benefit from the treatment. In this section, we discuss these subgroups and other related studies to define appropriate use of pertuzumab.

In the CLEOPATRA trial, the effect of pertuzumabbased treatment on PFS and overall survival (OS) was maintained in almost all subgroups investigated. ${ }^{40,41}$ However, the HR for OS in the non-visceral disease subgroup was 1.11 (95\% CI: 0.66-1.85). The authors noted that small patient populations, and data with wide confidence intervals limited the interpretation of data among patients in the subgroup with non-visceral disease. They also noted that a relatively small proportion of patients in this subgroup died (32.6\% overall) after a long survival time, with a median that was not reached in the pertuzumab group but was 61.5 months in the control group. On the other hand, HR of patients who received adjuvant or neoadjuvant trastuzumab treatment had similar PFS and OS ( 0.75 and 0.80 , respectively), although the number in this subgroup was also small.

In the APHINITY trial, ${ }^{42}$ preplanned subgroup analysis revealed that there was no difference between subgroups categorized by age. However, the number of invasive-disease events was low among patients with node-negative disease (32 of 897 patients in the pertuzumab group, and 29 of 902 patients in the placebo group). There was no detectable effect of the treatment (HR, $1.13 ; 95 \% \mathrm{CI}, 0.68-1.86 ; P=0.64)$. In the cohort of patients with node-positive disease, 139 patients $(9.2 \%)$ in the pertuzumab group and 181 patients $(12.1 \%)$ in the placebo group had invasive-disease events. The rate of survival from lack of invasive disease over 3 years was $92.0 \%$ in the pertuzumab group, and $90.2 \%$ in the placebo group (HR for an invasive disease event, 0.77 ; 95\% CI, 0.62 $0.96 ; P=0.02$ ). The lack of efficacy noted in node-negative disease was predicted to be a result of a low recurrence rate. ${ }^{74}$ Node-negative enrollment accounted for $36 \%$ of the patients in the APHINITY trial, which is higher than the $12 \%$ in the N9831 and B-31 trials which assessed the efficacy and safety of adding trastuzumab to paclitaxel followed by trastuzumab alone after completion of doxorubicin and cyclophosphamide chemotherapy, ${ }^{57}$ possibly diluting a potential benefit. This could have also accounted for an unexpected 93.2\% 3year DFS, compared to $86.7 \%$ 3.5-year DFS in the N9831 and B-31 trials, and approximately $90 \% 3$ to 5-year DFS in other trastuzumab trials. ${ }^{19,75,76}$ Excellent prognosis was also obtained in the APT trial. ${ }^{77}$ The 3-year rate of invasive disease-free survival was $98.7 \%$ (95\% CI: 97.6-99.8\%) for node-negative patients whose tumor size was no greater than
$3 \mathrm{~cm}$. Pertuzumab does not appear to be beneficial in patient with node-negative, small primary tumors.

In the NeoSphere trial, ${ }^{43}$ exploratory subgroup analyses suggested an association between total pCR (ypT0 with ypN0) and PFS when all treatment groups were combined. 5-year PFS rates were 85\% (95\% CI: 7691\%) for patients who achieved total pCR, compared with $76 \%$ (95\% CI: $71-81 \%)$ in patients who did not achieve total pCR (HR 0.54 [95\% CI: 0.29-1.00]). Results were consistent between hormone receptor-negative and positive disease. These results suggest that pertuzumab may not be effective for patients without visceral metastases in palliative settings, nor with nodal-negative, small primary tumor in adjuvant therapy.

\section{Treatment regimens}

In metastatic breast cancer patients, the duration of chemotherapy treatment periods directly associated with a detrimental impact on their quality of life (QoL). Metaanalysis found that longer durations of chemotherapy resulted in significant improvements in PFS and OS in patients with metastatic breast cancer. ${ }^{78}$ In contrast, a study of exploratory post hoc analysis of the CLEOPATRA trial data indicated that receiving more than 6 cycles of docetaxel was not associated with significant clinical benefits compared with the recommended minimum of 6 cycles (PFS HR $=0.80$, 95\% CI: $0.63-$ 1.01, $P=0.0640 ;$ OS $\mathrm{HR}=0.88,95 \% \mathrm{CI}: 0.69-1.12$, $P=0.3073){ }^{79}$ This exploratory analysis is limited by several factors, including moderate imbalances in patient characteristics between docetaxel treatment groups, small patient numbers in some subgroups, and the categorization of patients based on non-randomized factors. Therefore, these results should be interpreted with caution and confirmed in a randomized study.

In the PERUSE study, ${ }^{60}$ docetaxel was compared with paclitaxel and nab-paclitaxel. The median number of their treatment cycles was six, seven, and six, respectively. The safety profile of pertuzumab, trastuzumab, and taxane regimens in the PERUSE trial was generally consistent with results obtained in the CLEOPATRA trial. In these trials, the adverse events associated with the treatment regimens were diarrhea, alopecia, nausea, fatigue, and hematological toxicities. However, there was a notably lower incidence of neutropenia and febrile neutropenia in the PERUSE trial compared with the CLEOPATRA trial. This could be explained in part by the chemotherapy backbone, as incidences of febrile neutropenia were more similar between 
CLEOPATRA (14\%), and the docetaxel subgroup of PERUSE (11\%). The PFS and overall response rate were similar in these two trials, although we must be cautious about cross-trial comparisons. Other studies demonstrated the efficacy and tolerability of weekly paclitaxel treatment with pertuzumab and trastuzumab. ${ }^{80,81}$

Some groups selected other chemotherapeutic agents to use in combination with pertuzumab and trastuzumab. Eribulin and vinorelbine were investigated as the firstline therapy for metastatic or recurrent cancer. The SBCCSG-36 trial ${ }^{82}$ a small size, single arm, phase II study - demonstrated that the efficacy of eribulin-containing regimens was equivalent to that of the CLEOPATRA study. In a cohort of the VELVET trial, ${ }^{83,84}$ the overall response rate was broadly similar, but median PFS and duration of response were reduced when vinorelbine was used in combination with the two monoclonal antibodies, rather than docetaxel. The PHEREXA study ${ }^{85}$ showed that the median OS was increased by 8 months on using pertuzumab with trastuzumab plus capecitabine from trastuzumab and capecitabine group for second-line therapy, despite a modest increase in PFS. No new safety considerations were introduced after addition of pertuzumab to trastuzumab and capecitabine. The randomized, phase III neoadjuvant GeparSepto trial showed a significant increase from $29 \%$ to $38 \%(P=0.00065)$ in the proportion of patients achieving total pCR (ypT0 and ypN0) with weekly nab-paclitaxel. This treatment was compared to solubilized paclitaxel treatment (weekly) followed by epirubicin plus cyclophosphamide treatment in patients with primary, high-risk early breast cancer. ${ }^{86}$

In contrast, chemotherapy-free treatments may be effective yet safe for elderly or ineligible patients. The ongoing RESPECT trial ${ }^{87}$ is investigating the benefits of trastuzumab monotherapy, with respect to combination therapy of trastuzumab and chemotherapy in women over 70 years old with HER2-positive cancer, as a post-operative adjuvant therapy. The results from this trial may become a reference point for elderly or ineligible patients to choose a chemotherapy-free treatment with dual HER2 blockage. Further studies will result in the development of more alternative approaches for identifying patient-oriented chemotherapeutic agents that work with pertuzumab and trastuzumab.

Achieving pCR is remarkable in neoadjuvant therapy because most of the clinical trials concluded that pCR was associated with PFS. As mentioned in the efficacy section, insufficient therapeutic response rate increased in both study arms in the NeoSphere trials that did not complement the use of anti-HER2 antibodies with chemotherapy. The pCR rate in the pertuzumab and docetaxel study arm, and the pertuzumab and trastuzumab study arm was significantly lower than that of the pertuzumab, trastuzumab, and docetaxel arm. Therefore, chemotherapeutic agents and trastuzumab are beneficial in neoadjuvant therapy with pertuzumab. However, an optimal chemotherapy backbone for dual HER2 blockade in the neoadjuvant setting has not been identified.

Various treatment regimens have been attempted to resolve this issue. In the BERENICE trial, ${ }^{58}$ dose-dense anthracycline therapy was compared to traditional FEC therapy. In HER2-positive patients whose median age was 49.0 years, pCR rates were $61.8 \%$ in the study arm with dose-dense doxorubicin treatment plus cyclophosphamide that was followed up 2 weeks later by weekly paclitaxel injections (95\% CI: 54.7-68.6\%), and $60.7 \%$ in the study arm with FEC treatment followed by docetaxel $(95 \%$ CI: $53.6-67.5 \%)$. In the TRAIN-2 trial, ${ }^{72}$ anthracyclinefree regimens were evaluated for stage II or III patients. Similarly high proportions of pathological pCR were achieved with anthracyclines $(67 \%)$ or without anthracyclines $(68 \%)$. However, we have to consider that the patients in the non-anthracycline group received 9 cycles of paclitaxel $\left(80 \mathrm{mg} / \mathrm{m}^{2}\right.$ on days 1 and 8$)$, and carboplatin (area under the concentration-time curve [AUC] $6 \mathrm{mg} / \mathrm{mL}$ per min on day 1 , or AUC $3 \mathrm{mg} / \mathrm{mL}$ per min on days 1 and 8 , as per hospital preference). The KRISTINE trial $^{73,88}$ confirmed that in patients with HER2-positive stage II or III breast cancer, neoadjuvant treatment using docetaxel, carboplatin, and trastuzumab plus pertuzumab, was associated with a larger proportion of patients achieving pCR (56\%), and more patients receiving breast-conserving surgery than trastuzumab emtansine plus pertuzumab (44\%). However, compared with the chemotherapy-based regimen, trastuzumab emtansine plus pertuzumab was associated with fewer serious grade 3 or 4 adverse events, and longer maintenance of patient-reported health-related QoL and physical function. Selecting the chemotherapeutic backbone based on the patients' condition is essential.

\section{Biomarkers}

Initially, HER3 mRNA was predicted to be a biomarker for active ligand-induced HER2-HER3 signaling, with low HER3 mRNA levels correlating with clinical benefit from pertuzumab. ${ }^{89,90}$ However, a comprehensive prospective analysis of the biomarker data from the CLEOPATRA demonstrated that HER2 is the only marker 
suited for patient selection for the trastuzumab plus pertuzumab-based regimen in HER2-positive metastatic breast cancer. ${ }^{91}$ The same results were observed by an exploratory analysis of the TRYPHAENA trial, ${ }^{92}$ but the investigation revealed that lower $\mathrm{pCR}$ rates were observed in patients with tumors carrying PIK3CA mutations; however, this was not statistically significant. PIK3CA mutations correlated with a better prognosis in a series of untreated patients with HER2-positive cancer. ${ }^{93}$ Further exploratory studies are expected to validate biomarkers of pertuzumab-sensitive cancers.

From an immunological perspective, a retrospective analysis of the CLEOPATRA trial $^{94}$ revealed that increased number of stromal tumor-infiltrating lymphocytes (TILs) was significantly associated with improved OS in patients with HER2-positive metastatic breast cancer, treated with either pertuzumab or placebo combined with docetaxel and trastuzumab. However, it is still unclear how a combination of pertuzumab, trastuzumab, and docetaxel, is beneficial for the development of antitumor immunity. A group has reported that fucose was important for ADCC activity, and removal of the terminal sialic acid could enhance both ADCC (2 to 4-fold) and CDC (5-fold) activity of pertuzumab. ${ }^{95}$ Poorly sialylated pertuzumab can also lead to an increased clearance rate in mice, and co-injection with asialofetuin could protect the desialylated pertuzumab against asialoglycoprotein receptor-induced endocytosis in hepatocytes. ${ }^{95}$ Therefore, it is necessary to comprehensively examine not only the pharmacodynamics and contribution of the immune system, but also the pharmacokinetics of pertuzumab.

\section{Cost-effectiveness}

The above studies demonstrate the significant efficacy of pertuzumab. However, the pertuzumab treatment is quite expensive. Therefore, the cost-effectiveness of pertuzumab has been thoroughly discussed. The first study was reported in Canada ${ }^{96}$ comparing the cost-effectiveness of docetaxel, trastuzumab, and pertuzumab combination therapy for locally advanced, inflammatory, or early HER2positive breast cancer based on dual analyses of the NeoSphere trial and the TRYPHAENA trial. In this setting, pertuzumab was found to be more cost-effective, costing $\$ 25,388$ and $\$ 46,196$, respectively, per qualityadjusted life-year (QALY) gained. This group concluded that the addition of pertuzumab to neoadjuvant therapy is an attractive treatment option for HER2-positive early breast cancer patients. On the other hand, the incorporation of pertuzumab into a standard regimen of docetaxel and trastuzumab for treating metastatic HER2-overexpressing breast cancer was found not to provide enough benefit considering the cost of interventions generally deemed cost-effective in the United States. ${ }^{97}$ A similar result was published in Mexico. ${ }^{98}$ Another study suggested that incorporating pertuzumab into the available treatment regimens for HER2-positive early breast cancer was likely to be cost-effective for patients at a high risk of recurrence. ${ }^{99}$ Detailed analysis of the patient selection criteria in various clinical trials is necessary to ensure that prescribing pertuzumab is a cost-effective solution.

\section{For other HER2-positive cancers}

Some studies have tried to apply pertuzumab-containing regimen to HER2-overexpressing solid tumors. In MyPathway trial ${ }^{100}$ an ongoing, multicenter, phase IIa study that combines multiple basket studies under an adaptable master protocol-, objective responses were seen in nine primary tumor types: colorectal, bladder, biliary, salivary gland, pancreas, ovary, prostate, skin, and non-small-cell lung cancer. Trastuzumab in combination with chemotherapy improved OS of patients with HER2-positive advanced gastric or gastro-esophageal junction cancer in ToGA trial, ${ }^{101}$ therefore, pertuzumab was also expected the therapeutic effect for HER2-positive non-breast solid cancers. However, no clinical trials, including JACOB trial ${ }^{102}$ for metastatic gastric or gastroesophageal junction cancer and PENELOPE trial ${ }^{103}$ for platinum-resistant ovarian carcinoma with low tumor HER3 mRNA expression, were able to prove the benefit of pertuzumab yet. Further exploration of biomarkers or partner therapeutic agents will be needed for clinical application of pertuzumab to the treatment of non-breast solid tumors.

\section{Future directions}

Ongoing clinical trials of pertuzumab for breast cancer were listed in Table S1. Some groups tried to apply pertuzumab with atezolizumab, an anti-PD-L1 monoclonal antibody which prolonged PFS among patients with metastatic triple negative breast cancer. ${ }^{104}$ Connolly and colleagues showed that early changes in tumor maximum standardized uptake values corrected for lean body mass on [18F] fluorodeoxyglucose positron emission tomography/ computed tomography predicted response to four cycles of pertuzumab and trastuzumab therapy in estrogen receptornegative, HER2-positive breast cancer in the neoadjuvant 
setting. ${ }^{105}$ Results of these clinical trials will suggest more practical usage and more efficient selective methods of patients for pertuzumab-containing regimens.

\section{Conclusion}

Pertuzumab and trastuzumab bind to different sites on the HER2 receptor. Consequently, these two drugs achieve antitumor activity complementarily. Pertuzumab not only prolonged PFS for metastatic breast cancer patients, but also improved outcome of early breast cancer patients. However, the therapeutic benefit of pertuzumab is relatively small for patients with non-visceral metastases or with node-negative, small primary tumor. Moreover, we have to pay attention for cardiac dysfunction and diarrhea as the adverse effect. From the point view of risk-benefit balance and cost-effectiveness, it is important to discover new biomarkers and efficient regimens for pertuzumab by future studies.

\section{Disclosure}

Dr. Yamashiro reports honorariums from Chugai Pharmaceutical Co., Ltd., Kyowa Kirin Co., Ltd., AstraZeneca, Daiichi Sankyo Co., Ltd., Pfizer Japan Inc., and Novartis Pharma. The authors report no other conflicts of interest in this work.

\section{References}

1. Torre LA, Bray F, Siegel RL, Ferlay J, Lortet-Tieulent J, Jemal A. Global cancer statistics, 2012. CA Cancer J Clin. 2015;65(2):87-108. doi: $10.3322 /$ caac. 21262

2. Slamon DJ, Clark GM, Wong SG, Levin WJ, Ullrich A, McGuire WL. Human breast cancer: correlation of relapse and survival with amplification of the HER-2/neu oncogene. Science. 1987;235(4785):177182. doi:10.1126/science. 3798106

3. Ross JS, Slodkowska EA, Symmans WF, Pusztai L, Ravdin PM, Hortobagyi GN. The HER-2 receptor and breast cancer: ten years of targeted anti-HER-2 therapy and personalized medicine. Oncologist. 2009;14(4):320-368. doi:10.1634/theoncologist.2008-0230

4. Zell JA, Tsang WY, Taylor TH, Mehta RS, Anton-Culver H. Prognostic impact of human epidermal growth factor-like receptor 2 and hormone receptor status in inflammatory breast cancer (IBC): analysis of 2,014 IBC patient cases from the California cancer registry. Breast Cancer Res. 2009;11(1):R9. doi:10.1186/bcr2225

5. Rubin I, Yarden Y. The basic biology of HER2. Ann Oncol. 2001;12 (Suppl 1):S3-S8. doi:10.1093/annonc/12.suppl_1.s3

6. Loibl S, Gianni L. HER2-positive breast cancer. Lancet. 2017;389 (10087):2415-2429. doi:10.1016/S0140-6736(16)32417-5

7. Yarden Y, Sliwkowski MX. Untangling the ErbB signalling network. Nat Rev Mol Cell Biol. 2001;2(2):127-137. doi:10.1038/35052073

8. Hynes NE, Lane HA. ERBB receptors and cancer: the complexity of targeted inhibitors. Nat Rev Cancer. 2005;5(5):341-354. doi:10.1038/ nrc1609

9. Klapper LN, Kirschbaum MH, Sela M, Yarden Y. Biochemical and clinical implications of the ErbB/HER signaling network of growth factor receptors. Adv Cancer Res. 2000;77:25-79.
10. Pinkas-Kramarski R, Soussan L, Waterman $\mathrm{H}$, et al. Diversification of neu differentiation factor and epidermal growth factor signaling by combinatorial receptor interactions. Embo J. 1996;15(10):2452-2467. doi:10.1200/JCO.2013.53.9 288

11. Tzahar E, Waterman H, Chen X, et al. A hierarchical network of interreceptor interactions determines signal transduction by neu differentiation factor/neuregulin and epidermal growth factor. $\mathrm{Mol}$ Cell Biol. 1996;16(10):5276-5287. doi:10.1128/mcb.16.10.5276

12. Citri A, Skaria KB, Yarden Y. The deaf and the dumb: the biology of ErbB-2 and ErbB-3. Exp Cell Res. 2003;284(1):54-65. doi:10.1016/s0014-4827(02)00101-5

13. Baselga J, Tripathy D, Mendelsohn J, et al. Phase II study of weekly intravenous recombinant humanized anti-p185HER2 monoclonal antibody in patients with HER2/neu-overexpressing metastatic breast cancer. J Clin Oncol. 1996;14(3):737-744. doi:10.1200/JCO.1996.14.3.737

14. Ghosh R, Narasanna A, Wang SE, et al. Trastuzumab has preferential activity against breast cancers driven by HER2 homodimers. Cancer Res. 2011;71(5):1871-1882. doi:10.1158/0008-5472.CAN-10-1872

15. Junttila TT, Akita RW, Parsons K, et al. Ligand-independent HER2/HER3/PI3K complex is disrupted by trastuzumab and is effectively inhibited by the PI3K inhibitor GDC-0941. Cancer Cell. 2009;15(5):429-440. doi:10.1016/j.ccr.2009.03.020

16. Molina MA, Codony-Servat J, Albanell J, Rojo F, Arribas J, Baselga J. Trastuzumab (Herceptin), a humanized anti-Her2 receptor monoclonal antibody, inhibits basal and activated Her2 ectodomain cleavage in breast cancer cells. Cancer Res. 2001;61(12):4744-4749.

17. Nahta R, Esteva FJ. Herceptin: mechanisms of action and resistance. Cancer Lett. 2006;232(2):123-138. doi:10.1016/j.canlet.2005.01.041

18. Hudis CA. Trastuzumab-mechanism of action and use in clinical practice. $N$ Engl $J$ Med. 2007;357(1):39-51. doi:10.1056/ NEJMra043186

19. Slamon D, Eiermann W, Robert N, et al. Adjuvant trastuzumab in HER2-positive breast cancer. N Engl J Med. 2011;365(14):12731283. doi:10.1056/NEJMoa0910383

20. Gianni L, Pienkowski T, Im YH, et al. Efficacy and safety of neoadjuvant pertuzumab and trastuzumab in women with locally advanced, inflammatory, or early HER2-positive breast cancer (NeoSphere): a randomised multicentre, open-label, phase 2 trial. Lancet Oncol. 2012;13(1):25-32. doi:10.1016/S1470-2045(11)70336-9

21. Slamon DJ, Leyland-Jones B, Shak S, et al. Use of chemotherapy plus a monoclonal antibody against HER2 for metastatic breast cancer that overexpresses HER2. N Engl J Med. 2001;344 (11):783-792. doi:10.1056/NEJM200103153441101

22. Kumler I, Tuxen MK, Nielsen DL. A systematic review of dual targeting in HER2-positive breast cancer. Cancer Treat Rev. 2014;40(2):259-270. doi:10.1016/j.ctrv.2013.09.002

23. Garrett JT, Arteaga CL. Resistance to HER2-directed antibodies and tyrosine kinase inhibitors: mechanisms and clinical implications. Cancer Biol Ther. 2011;11(9):793-800. doi:10.4161/cbt.11. 9.15045

24. Jin MH, Nam AR, Park JE, Bang JH, Bang YJ, Oh DY. Resistance mechanism against trastuzumab in HER2-positive cancer cells and its negation by Src inhibition. Mol Cancer Ther. 2017;16(6):11451154. doi:10.1158/1535-7163.MCT-16-0669

25. Luque-Cabal M, Garcia-Teijido P, Fernandez-Perez Y, SanchezLorenzo L, Palacio-Vazquez I. Mechanisms behind the resistance to trastuzumab in HER2-amplified breast cancer and strategies to overcome it. Clin Med Insights Oncol. 2016;10(Suppl 1):21-30. doi: $10.4137 / \mathrm{CMO} . \mathrm{S} 34537$

26. Li Y, Chu J, Feng W, et al. EPHA5 mediates trastuzumab resistance in HER2-positive breast cancers through regulating cancer stem cell-like properties. Faseb J. 2019;33(4):4851-4865. doi:10.1096/fj.201701561RRRR 
27. Richard S, Selle F, Lotz JP, Khalil A, Gligorov J, Soares DG. Pertuzumab and trastuzumab: the rationale way to synergy. $A n$ Acad Bras Cienc. 2016;88(Suppl 1):565-577. doi:10.1590/00013765201620150178

28. Agus DB, Akita RW, Fox WD, et al. Targeting ligand-activated ErbB2 signaling inhibits breast and prostate tumor growth. Cancer Cell. 2002;2(2):127-137.

29. Nahta R, Hung MC, Esteva FJ. The HER-2-targeting antibodies trastuzumab and pertuzumab synergistically inhibit the survival of breast cancer cells. Cancer Res. 2004;64(7):2343-2346.

30. Metzger-Filho O, Winer EP, Krop I. Pertuzumab: Optimizing HER2 blockade. Clin Cancer Res. 2013;19(20):5552-5556. doi:10.1158/1078-0432.CCR-13-0518

31. Nahta R, Yuan LX, Zhang B, Kobayashi R, Esteva FJ. Insulinlike growth factor-I receptor/human epidermal growth factor receptor 2 heterodimerization contributes to trastuzumab resistance of breast cancer cells. Cancer Res. 2005;65(23):11118 11128. doi:10.1158/0008-5472.CAN-04-3841

32. Lee-Hoeflich ST, Crocker L, Yao E, et al. A central role for HER3 in HER2-amplified breast cancer: implications for targeted therapy. Cancer Res. 2008;68(14):5878-5887. doi:10.1158/00085472.CAN-08-0380

33. Sergina NV, Rausch M, Wang D, et al. Escape from HER-family tyrosine kinase inhibitor therapy by the kinase-inactive HER3. Nature. 2007;445(7126):437-441. doi:10.1038/nature05474

34. Amin DN, Sergina N, Ahuja D, et al. Resiliency and vulnerability in the HER2-HER3 tumorigenic driver. Sci Transl Med. 2010;2 (16):16ra7. doi:10.1126/scitranslmed.3000389

35. Garrett JT, Olivares MG, Rinehart C, et al. Transcriptional and posttranslational up-regulation of HER3 (ErbB3) compensates for inhibition of the HER2 tyrosine kinase. Proc Natl Acad Sci U S A. 2011;108(12):5021-5026. doi:10.1073/pnas.1016140108

36. Scheuer W, Friess T, Burtscher H, Bossenmaier B, Endl J, Hasmann M. Strongly enhanced antitumor activity of trastuzumab and pertuzumab combination treatment on HER2-positive human xenograft tumor models. Cancer Res. 2009;69(24):9330-9336. doi:10.1158/0008-5472.CAN-08-4597

37. Mamidi S, Cinci M, Hasmann M, Fehring V, Kirschfink M Lipoplex mediated silencing of membrane regulators (CD46, CD55 and CD59) enhances complement-dependent anti-tumor activity of trastuzumab and pertuzumab. Mol Oncol. 2013;7 (3):580-594. doi:10.1016/j.molonc.2013.02.011

38. Baselga J, Gelmon KA, Verma S, et al. Phase II trial of pertuzumab and trastuzumab in patients with human epidermal growth factor receptor 2-positive metastatic breast cancer that progressed during prior trastuzumab therapy. J Clin Oncol. 2010;28(7):11381144. doi: $10.1200 / J C O .2009 .24 .2024$

39. Baselga J, Cortes J, Kim SB, et al. Pertuzumab plus trastuzumab plus docetaxel for metastatic breast cancer. $N$ Engl $J$ Med 2012;366(2):109-119. doi:10.1056/NEJMoa1113216

40. Swain SM, Kim SB, Cortes J, et al. Pertuzumab, trastuzumab, and docetaxel for HER2-positive metastatic breast cancer (CLEOPATRA study): overall survival results from a randomised, double-blind, placebo-controlled, phase 3 study. Lancet Oncol. 2013;14(6):461-471. doi:10.1016/S1470-2045(13)70130-X

41. Swain SM, Baselga J, Kim SB, et al. Pertuzumab, trastuzumab, and docetaxel in HER2-positive metastatic breast cancer. $N$ Engl $J$ Med. 2015;372(8):724-734. doi:10.1056/NEJMoa1413513

42. von Minckwitz G, Procter M, de Azambuja E, et al. Adjuvant pertuzumab and trastuzumab in early HER2-positive breast cancer. $N$ Engl J Med. 2017;377(2):122-131. doi:10.1056/NEJMoa1703643

43. Gianni L, Pienkowski T, Im YH, et al. 5-year analysis of neoadjuvant pertuzumab and trastuzumab in patients with locally advanced, inflammatory, or early-stage HER2-positive breast cancer (NeoSphere): a multicentre, open-label, phase 2 randomised trial. Lancet Oncol. 2016;17(6):791-800. doi:10.1016/S1470-2045(16)00163-7
44. Schneeweiss A, Chia S, Hickish T, et al. Pertuzumab plus trastuzumab in combination with standard neoadjuvant anthracyclinecontaining and anthracycline-free chemotherapy regimens in patients with HER2-positive early breast cancer: a randomized phase II cardiac safety study (TRYPHAENA). Ann Oncol. 2013;24(9):2278-2284. doi:10.1093/annonc/mdt182

45. Schneeweiss A, Chia S, Hickish T, et al. Long-term efficacy analysis of the randomised, phase II TRYPHAENA cardiac safety study: evaluating pertuzumab and trastuzumab plus standard neoadjuvant anthracycline-containing and anthracycline-free chemotherapy regimens in patients with HER2-positive early breast cancer. Eur $J$ Cancer. 2018;89:27-35. doi:10.1016/j.ejca.2017.10.021

46. Portera CC, Walshe JM, Rosing DR, et al. Cardiac toxicity and efficacy of trastuzumab combined with pertuzumab in patients with [corrected] human epidermal growth factor receptor 2-positive metastatic breast cancer. Clin Cancer Res. 2008;14(9):27102716. doi:10.1158/1078-0432.CCR-07-4636

47. Lee KF, Simon H, Chen H, Bates B, Hung MC, Hauser C. Requirement for neuregulin receptor erbB2 in neural and cardiac development. Nature. 1995;378(6555):394-398. doi:10.1038/ 378394a0

48. Gassmann M, Casagranda F, Orioli D, et al. Aberrant neural and cardiac development in mice lacking the ErbB4 neuregulin receptor. Nature. 1995;378(6555):390-394

49. Crone SA, Zhao YY, Fan L, et al. ErbB2 is essential in the prevention of dilated cardiomyopathy. Nat Med. 2002;8(5):459465. doi: 10.1038/nm0502-459

50. Ozcelik C, Erdmann B, Pilz B, et al. Conditional mutation of the ErbB2 (HER2) receptor in cardiomyocytes leads to dilated cardiomyopathy. Proc Natl Acad Sci U S A. 2002;99(13):8880-8885. doi:10.1073/pnas.122249299

51. Zhao YY, Sawyer DR, Baliga RR, et al. Neuregulins promote survival and growth of cardiac myocytes. persistence of ErbB2 and ErbB4 expression in neonatal and adult ventricular myocytes. J Biol Chem. 1998;273(17):10261-10269. doi:10.1074/jbc.273. 17.10261

52. Sendur MA, Aksoy S, Altundag K. Pertuzumab-induced cardiotoxicity: safety compared with trastuzumab. Future Oncol. 2015;11(1):13-15. doi:10.2217/fon.14.184

53. Seidman A, Hudis C, Pierri MK, et al. Cardiac dysfunction in the trastuzumab clinical trials experience. J Clin Oncol. 2002;20 (5):1215-1221. doi:10.1200/JCO.2002.20.5.1215

54. Piccart-Gebhart MJ, Procter M, Leyland-Jones B, et al. Trastuzumab after adjuvant chemotherapy in HER2-positive breast cancer. $N$ Engl J Med. 2005;353(16):1659-1672. doi:10.1056/ NEJMoa052306

55. Romond EH, Perez EA, Bryant J, et al. Trastuzumab plus adjuvant chemotherapy for operable HER2-positive breast cancer. $N$ Engl J Med. 2005;353(16):1673-1684. doi:10.1056/NEJMoa0 52122

56. de Azambuja E, Procter MJ, van Veldhuisen DJ, et al. Trastuzumab-associated cardiac events at 8 years of median follow-up in the Herceptin adjuvant trial (BIG 1-01). J Clin Oncol. 2014;32(20):2159-2165. doi:10.1200/JCO.2013.53.9288

57. Perez EA, Romond EH, Suman VJ, et al. Trastuzumab plus adjuvant chemotherapy for human epidermal growth factor receptor 2-positive breast cancer: planned joint analysis of overall survival from NSABP B-31 and NCCTG N9831. J Clin Oncol. 2014;32(33):3744-3752. doi:10.1200/JCO.2014.55.5730

58. Swain SM, Ewer MS, Viale G, et al. Pertuzumab, trastuzumab, and standard anthracycline- and taxane-based chemotherapy for the neoadjuvant treatment of patients with HER2-positive localized breast cancer (BERENICE): a phase II, open-label, multicenter, multinational cardiac safety study. Ann Oncol. 2018;29 (3):646-653. doi:10.1093/annonc/mdx773 
59. Yu AF, Singh JC, Wang R, et al. Cardiac safety of dual anti-HER2 therapy in the neoadjuvant setting for treatment of HER2-positive breast cancer. Oncologist. 2017;22(6):642-647. doi:10.1634/ theoncologist.2016-0406

60. Bachelot T, Ciruelos E, Schneeweiss A, et al. Preliminary safety and efficacy of first-line pertuzumab combined with trastuzumab and taxane therapy for HER2-positive locally recurrent or metastatic breast cancer (PERUSE). Ann Oncol. 2019. doi:10.1016/ S0140-6736(13)61094-6

61. De Lorenzo C, Paciello R, Riccio G, et al. Cardiotoxic effects of the novel approved anti-ErbB2 agents and reverse cardioprotective effects of ranolazine. Onco Targets Ther. 2018;11:22412250. doi:10.2147/OTT.S157294

62. Perjeta ${ }^{\circledR}$ (pertuzumab) injection, for intravenous use [package insert]. San Francisco, CA: Genentech, Inc; 2018.

63. Zhu C, Ling W, Zhang J, Gao H, Shen K, Ma X. Safety and efficacy evaluation of pertuzumab in patients with solid tumors. Medicine (Baltimore). 2017;96(20):e6870. doi:10.1097/MD.00 00000000006870

64. Van Sebille YZ, Gibson RJ, Wardill HR, Bowen JM. ErbB small molecule tyrosine kinase inhibitor (TKI) induced diarrhoea: chloride secretion as a mechanistic hypothesis. Cancer Treat Rev. 2015;41(7):646-652. doi:10.1016/j. ctrv.2015.05.011

65. Melosky B. Supportive care treatments for toxicities of antiEGFR and other targeted agents. Curr Oncol. 2012;19(Suppl 1): S59-S63. doi:10.3747/co.19.1054

66. Gibson RJ, Keefe DM. Cancer chemotherapy-induced diarrhoea and constipation: mechanisms of damage and prevention strategies. Support Care Cancer. 2006;14(9):890-900. doi:10.1007/ s00520-006-0040-y

67. Swain SM, Schneeweiss A, Gianni L, et al. Incidence and management of diarrhea in patients with HER2-positive breast cancer treated with pertuzumab. Ann Oncol. 2017;28(4):761-768. doi:10.1093/annonc/mdw695

68. Hussain N, Said ASA, Khan Z. Safety assessment of neoadjuvant pertuzumab combined with trastuzumab in nonmetastatic HER2positive breast cancer in postmenopausal elderly women of South Asia. Int J Breast Cancer. 2018;2018:6106041. doi:10.1155/2018/ 6106041

69. Wu PE, Juurlink DN. Clinical review: loperamide toxicity. Ann Emerg Med. 2017;70(2):245-252. doi:10.1016/j.annemergmed. 2017.04.008

70. Gao JJ, Tan M, Pohlmann PR, Swain SM. HALT-D: a phase II evaluation of crofelemer for the prevention and prophylaxis of diarrhea in patients with breast cancer on pertuzumab-based regimens. Clin Breast Cancer. 2017;17(1):76-78. doi:10.1016/j. clbc.2016.08.005

71. Squires H, Pandor A, Thokala P, et al. Pertuzumab for the neoadjuvant treatment of early-stage HER2-positive breast cancer: an evidence review group perspective of a NICE single technology appraisal. Pharmacoeconomics. 2018;36(1):29-38. doi:10.1007/ s40273-017-0556-7

72. van Ramshorst MS, van der Voort A, van Werkhoven ED, et al. Neoadjuvant chemotherapy with or without anthracyclines in the presence of dual HER2 blockade for HER2-positive breast cancer (TRAIN-2): a multicentre, open-label, randomised, phase 3 trial. Lancet Oncol. 2018;19(12):1630-1640. doi:10.1016/S1470-2045 (18)30570-9

73. Hurvitz SA, Martin M, Symmans WF, et al. Neoadjuvant trastuzumab, pertuzumab, and chemotherapy versus trastuzumab emtansine plus pertuzumab in patients with HER2-positive breast cancer (KRISTINE): a randomised, open-label, multicentre, phase 3 trial. Lancet Oncol. 2018;19(1):115-126. doi:10.1016/S14702045(17)30716-7
74. Bulbul A, Araujo-Mino E, Dayao ZR. The conundrum of adjuvant HER2 treatment options. Front Oncol. 2018;8:177. doi: $10.3389 /$ fonc. 2018.00177

75. Goldhirsch A, Gelber RD, Piccart-Gebhart MJ, et al. 2 years versus 1 year of adjuvant trastuzumab for HER2-positive breast cancer (HERA): an open-label, randomised controlled trial. Lancet. 2013;382(9897):1021-1028. doi:10.1016/S0140-6736 (13)61094-6

76. Chan A, Delaloge S, Holmes FA, et al. Neratinib after trastuzumab-based adjuvant therapy in patients with HER2-positive breast cancer (ExteNET): a multicentre, randomised, doubleblind, placebo-controlled, phase 3 trial. Lancet Oncol. 2016;17 (3):367-377. doi:10.1016/S1470-2045(15)00551-3

77. Tolaney SM, Barry WT, Dang CT, et al. Adjuvant paclitaxel and trastuzumab for node-negative, HER2-positive breast cancer. $N$ Engl J Med. 2015;372(2):134-141. doi:10.1056/NEJMoa1406281

78. Gennari A, Stockler M, Puntoni M, et al. Duration of chemotherapy for metastatic breast cancer: a systematic review and metaanalysis of randomized clinical trials. J Clin Oncol. 2011;29 (16):2144-2149. doi:10.1200/JCO.2010.31.5374

79. Miles D, Im YH, Fung A, et al. Effect of docetaxel duration on clinical outcomes: exploratory analysis of CLEOPATRA, a phase III randomized controlled trial. Ann Oncol. 2017;28(11):27612767. doi:10.1093/annonc/mdx406

80. Dang C, Iyengar N, Datko F, et al. Phase II study of paclitaxel given once per week along with trastuzumab and pertuzumab in patients with human epidermal growth factor receptor 2-positive metastatic breast cancer. J Clin Oncol. 2015;33(5):442-447. doi:10.1200/JCO.2014.57.1745

81. Smyth LM, Iyengar NM, Chen MF, et al. Weekly paclitaxel with trastuzumab and pertuzumab in patients with HER2-overexpressing metastatic breast cancer: overall survival and updated progression-free survival results from a phase II study. Breast Cancer Res Treat. 2016;158(1):91-97. doi:10.1007/s10549-016-3851-7

82. Inoue $\mathrm{K}$, Ninomiya $\mathrm{J}$, Saito $\mathrm{T}$, et al. Eribulin, trastuzumab, and pertuzumab as first-line therapy for patients with HER2-positive metastatic breast cancer: a phase II, multicenter, collaborative, open-label, single-arm clinical trial. Invest New Drugs. 2019;37 (3):538-547. doi:10.1007/s10637-019-00755-x

83. Andersson M, Lopez-Vega JM, Petit T, et al. Efficacy and safety of pertuzumab and trastuzumab administered in a single infusion bag, followed by vinorelbine: VELVET cohort 2 final results. Oncologist. 2017;22(10):1160-1168. doi:10.1634/theoncologist. 2017-0079

84. Perez EA, Lopez-Vega JM, Petit T, et al. Safety and efficacy of vinorelbine in combination with pertuzumab and trastuzumab for first-line treatment of patients with HER2-positive locally advanced or metastatic breast cancer: VELVET cohort 1 final results. Breast Cancer Res. 2016;18(1):126-016-0773-6. doi:10.1186/s13058-0160773-6

85. Urruticoechea A, Rizwanullah M, Im SA, et al. Randomized phase III trial of trastuzumab plus capecitabine with or without pertuzumab in patients with human epidermal growth factor receptor 2-positive metastatic breast cancer who experienced disease progression during or after trastuzumab-based therapy. J Clin Oncol. 2017;35(26):3030-3038. doi:10.1200/JCO.2016.70.6267

86. Untch M, Jackisch C, Schneeweiss A, et al. Nab-paclitaxel versus solvent-based paclitaxel in neoadjuvant chemotherapy for early breast cancer (GeparSepto-GBG 69): A randomised, phase 3 trial. Lancet Oncol. 2016;17(3):345-356. doi:10.1016/S1470-2045(15) 00542-2

87. Sawaki M, Tokudome N, Mizuno T, et al. Evaluation of trastuzumab without chemotherapy as a post-operative adjuvant therapy in HER2-positive elderly breast cancer patients: randomized controlled trial [RESPECT (N-SAS BC07). Jpn J Clin Oncol. 2011;41(5):709-712. doi:10.1093/jjco/hyr011 
88. Okines AF. T-DM1 in the neo-adjuvant treatment of HER2-positive breast cancer: impact of the KRISTINE (TRIO-021) trial. Rev Recent Clin Trials. 2017;12(3):216-222. doi:10.2174/157488 7112666170529094911

89. Amler LC. HER3 mRNA as a predictive biomarker in anticancer therapy. Expert Opin Biol Ther. 2010;10(9):1343-1355. doi:10.15 17/14712598.2010.512003

90. Thomas G, Chardes T, Gaborit N, et al. HER3 as biomarker and therapeutic target in pancreatic cancer: new insights in pertuzumab therapy in preclinical models. Oncotarget. 2014;5(16):71387148. doi:10.18632/oncotarget.2231

91. Baselga J, Cortes J, Im SA, et al. Biomarker analyses in CLEOPATRA: A phase III, placebo-controlled study of pertuzumab in human epidermal growth factor receptor 2-positive, firstline metastatic breast cancer. J Clin Oncol. 2014;32(33):37533761. doi:10.1200/JCO.2013.54.5384

92. Schneeweiss A, Chia S, Hegg R, et al. Evaluating the predictive value of biomarkers for efficacy outcomes in response to pertuzumab- and trastuzumab-based therapy: an exploratory analysis of the TRYPHAENA study. Breast Cancer Res. 2014;16(4):R73. doi:10.1186/s13058-014-0492-9

93. Cizkova M, Susini A, Vacher S, et al. PIK3CA mutation impact on survival in breast cancer patients and in ERalpha, PR and ERBB2-based subgroups. Breast Cancer Res. 2012;14(1):R28. doi:10.1186/bcr3169

94. Luen SJ, Salgado R, Fox S, et al. Tumour-infiltrating lymphocytes in advanced HER2-positive breast cancer treated with pertuzumab or placebo in addition to trastuzumab and docetaxel: a retrospective analysis of the CLEOPATRA study. Lancet Oncol. 2017;18 (1):52-62. doi:10.1016/S1470-2045(16)30631-3

95. Luo $\mathrm{C}$, Chen $\mathrm{S}, \mathrm{Xu} \mathrm{N}$, et al. Glycoengineering of pertuzumab and its impact on the pharmacokinetic/pharmacodynamic properties. Sci Rep. 2017;7:46347. doi:10.1038/srep46347

96. Attard CL, Pepper AN, Brown ST, et al. Cost-effectiveness analysis of neoadjuvant pertuzumab and trastuzumab therapy for locally advanced, inflammatory, or early HER2-positive breast cancer in Canada. J Med Econ. 2015;18(3):173-188. doi:10.3111/136 96998.2014.979938

97. Durkee BY, Qian Y, Pollom EL, et al. Cost-effectiveness of pertuzumab in human epidermal growth factor receptor 2-positive metastatic breast cancer. J Clin Oncol. 2016;34(9):902-909. doi: $10.1200 /$ JCO.2015.62.9105
98. Diaby V, Ali AA, Williams KJ, et al. Economic evaluation of sequencing strategies in HER2-positive metastatic breast cancer in Mexico: a contrast between public and private payer perspectives. Breast Cancer Res Treat. 2017;166(3):951-963. doi:10.1007/s10549-017-4473-4

99. Garrison LP Jr, Babigumira J, Tournier C, Goertz HP, Lubinga SJ, Perez EA. Cost-effectiveness analysis of pertuzumab with trastuzumab and chemotherapy compared to trastuzumab and chemotherapy in the adjuvant treatment of HER2-positive breast cancer in the united states. Value Health. 2019;22(4):408-415. doi:10.1016/j.jval.2018.11.014

100. Hainsworth JD, Meric-Bernstam F, Swanton C, et al. Targeted therapy for advanced solid tumors on the basis of molecular profiles: results from MyPathway, an open-label, phase IIa multiple basket study. J Clin Oncol. 2018;36(6):536-542. doi:10.1200/ JCO.2017.75.3780

101. Bang YJ, Van Cutsem E, Feyereislova A, et al. Trastuzumab in combination with chemotherapy versus chemotherapy alone for treatment of HER2-positive advanced gastric or gastro-oesophageal junction cancer (ToGA): a phase 3, open-label, randomised controlled trial. Lancet. 2010;376(9742):687-697. doi:10.1016/ S0140-6736(10)61121-X

102. Tabernero J, Hoff PM, Shen L, et al. Pertuzumab plus trastuzumab and chemotherapy for HER2-positive metastatic gastric or gastro-oesophageal junction cancer (JACOB): final analysis of a double-blind, randomised, placebo-controlled phase 3 study. Lancet Oncol. 2018;19(10):1372-1384. doi:10.1016/S1470-2045 (18)30481-9

103. Kurzeder C, Bover I, Marme F, et al. Double-blind, placebocontrolled, randomized phase III trial evaluating pertuzumab combined with chemotherapy for low tumor human epidermal growth factor receptor 3 mRNA-expressing platinum-resistant ovarian cancer (PENELOPE). J Clin Oncol. 2016;34(21):25162525. doi: $10.1200 / J C O .2015 .66 .0787$

104. Schmid P, Adams S, Rugo HS, et al. Atezolizumab and nabpaclitaxel in advanced triple-negative breast cancer. $N$ Engl $J$ Med. 2018;379(22):2108-2121. doi:10.1056/NEJMoa1809615

105. Connolly RM, Leal JP, Solnes L, et al. TBCRC026: phase II trial correlating standardized uptake value with pathologic complete response to pertuzumab and trastuzumab in breast cancer. J Clin Oncol. 2019;37(9):714-722. doi:10.1200/ JCO.2018.78.7986 


\begin{tabular}{|c|c|c|c|c|c|c|c|c|c|c|c|c|c|c|c|c|c|c|c|c|c|c|c|c|}
\hline 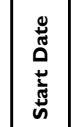 & 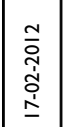 & 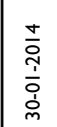 & 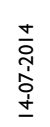 & 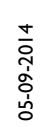 & 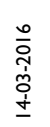 & 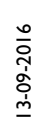 & 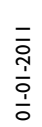 & 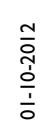 & $\begin{array}{l}\frac{m}{o} \\
\stackrel{0}{0} \\
\frac{1}{0}\end{array}$ & 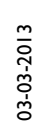 & 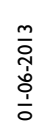 & 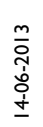 & 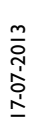 & $\begin{array}{l}\frac{m}{\grave{i}} \\
\stackrel{1}{0} \\
\frac{1}{0}\end{array}$ & $\begin{array}{l}\frac{m}{\grave{j}} \\
\text { ò } \\
\frac{1}{0}\end{array}$ & 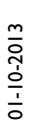 & 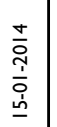 & $\begin{array}{l}\frac{v}{a} \\
\frac{T}{\bar{q}} \\
\frac{\underline{m}}{m}\end{array}$ & 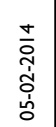 & 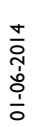 & 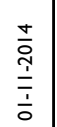 & 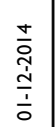 & 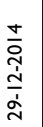 & 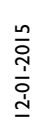 \\
\hline
\end{tabular}

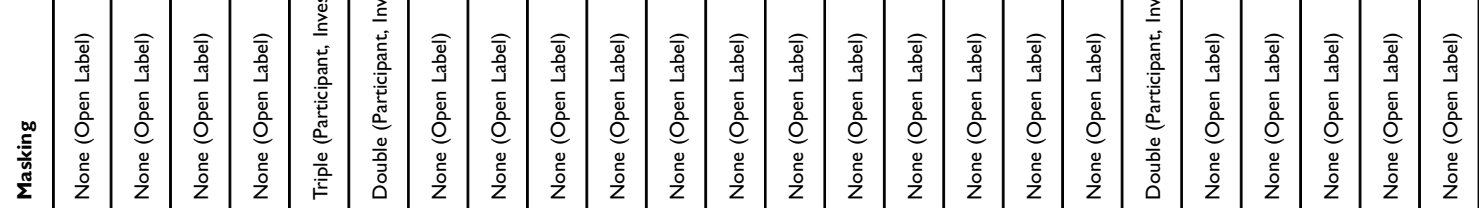

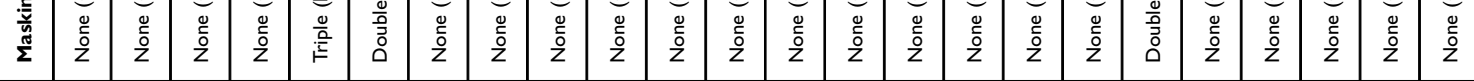

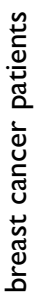

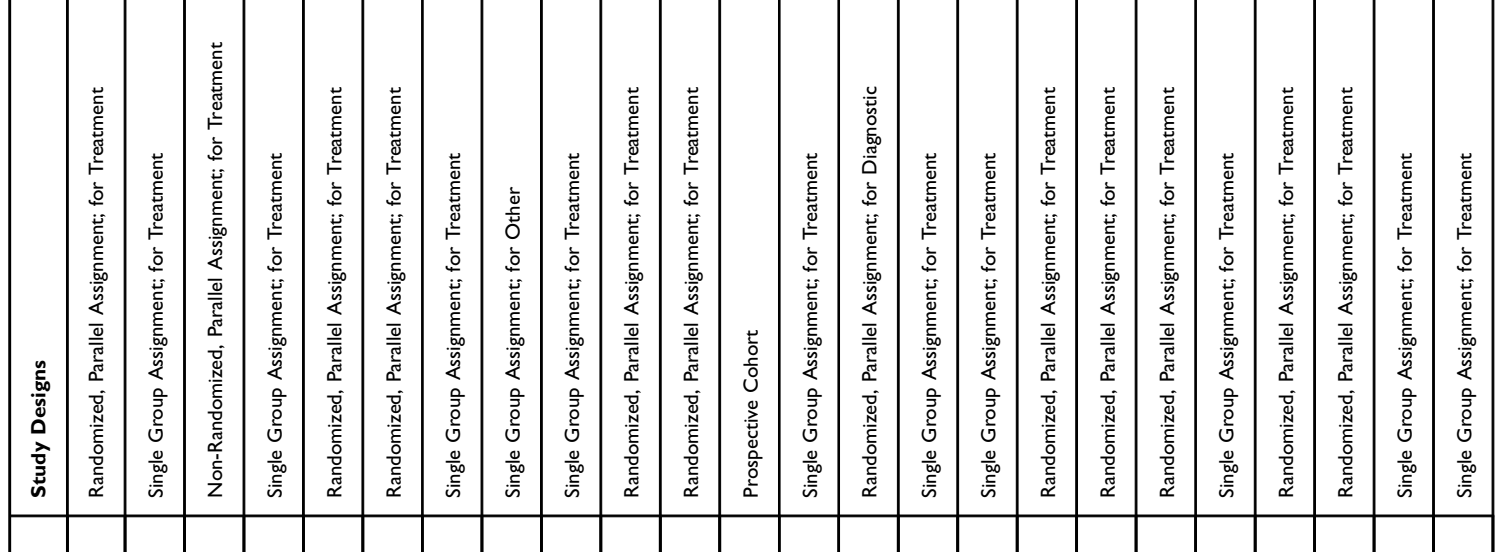

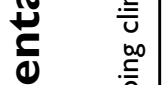

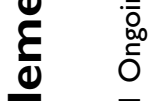

$\begin{array}{ll}\frac{1}{2} & \bar{n} \\ \frac{1}{n} & \frac{0}{0}\end{array}$

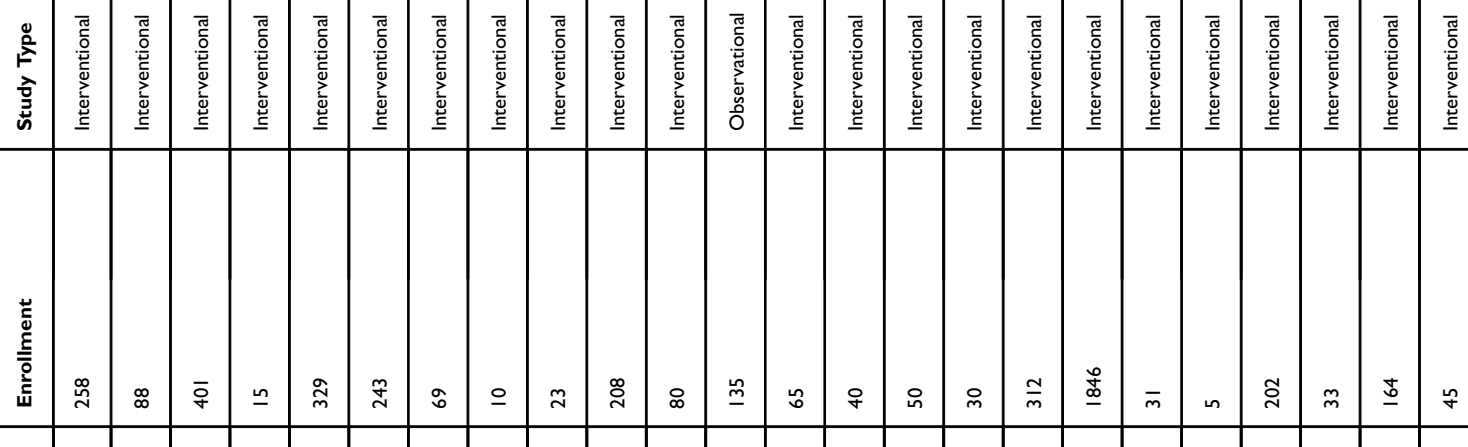

党 


\begin{tabular}{|c|c|c|c|c|c|c|c|c|c|c|c|c|c|c|c|c|c|c|c|c|c|c|c|c|c|}
\hline 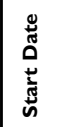 & 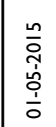 & $\begin{array}{l}\stackrel{n}{\dot{T}} \\
\underline{\dot{I}} \\
\underline{\dot{\omega}}\end{array}$ & $\begin{array}{l}\frac{n}{\grave{i}} \\
\frac{\grave{I}}{\dot{m}}\end{array}$ & 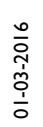 & $\begin{array}{l}\frac{0}{\bar{a}} \\
\frac{1}{0} \\
\frac{1}{0}\end{array}$ & 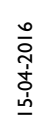 & 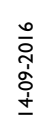 & $\begin{array}{l}\hat{\bar{T}} \\
\stackrel{\tilde{D}}{0} \\
\underline{M}\end{array}$ & 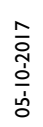 & $\begin{array}{l}\frac{\infty}{\grave{3}} \\
\dot{\phi}+ \\
\dot{+}\end{array}$ & $\begin{array}{l}\frac{a}{0} \\
\hat{d} \\
\frac{\hat{\sigma}}{0} \\
\frac{9}{0}\end{array}$ & 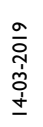 & 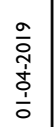 & $\begin{array}{l}\frac{a}{0} \\
\frac{1}{0} \\
\frac{1}{0}\end{array}$ & 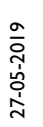 & 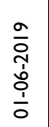 & 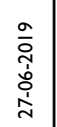 & $\begin{array}{l}\frac{a}{\bar{T}} \\
\stackrel{1}{0} \\
\frac{\grave{o}}{0}\end{array}$ & $\begin{array}{l}\frac{a}{\bar{T}} \\
\hat{1} \\
\frac{1}{0}\end{array}$ & 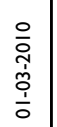 & 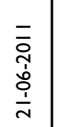 & 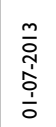 & 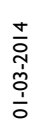 & 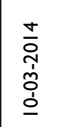 & $\begin{array}{l}\frac{v}{9} \\
\frac{i}{\bar{T}} \\
\frac{1}{0}\end{array}$ \\
\hline
\end{tabular}

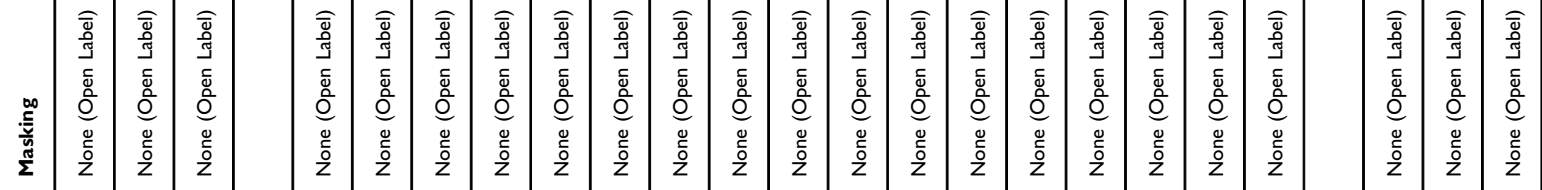

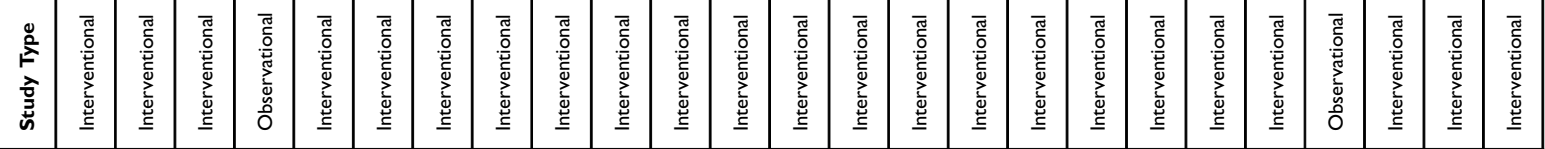

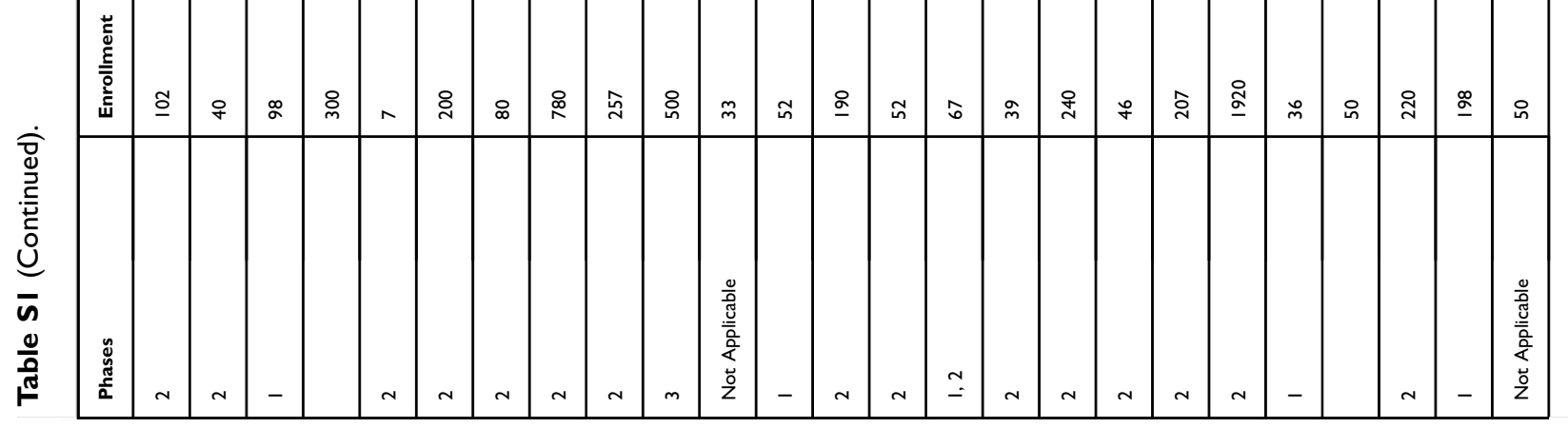




\begin{tabular}{|c|c|c|c|c|c|c|c|c|c|c|c|c|c|c|c|c|c|c|c|c|c|c|c|c|c|}
\hline 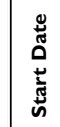 & 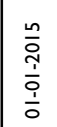 & 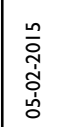 & $\begin{array}{l}\frac{n}{2} \\
\frac{T}{+} \\
\frac{1}{0}\end{array}$ & $\begin{array}{l}\frac{n}{2} \\
\frac{T}{0} \\
\frac{9}{0}\end{array}$ & 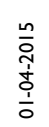 & 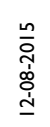 & 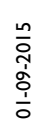 & $\begin{array}{l}\frac{n}{\grave{T}} \\
\frac{i}{\grave{1}}\end{array}$ & 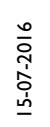 & $\begin{array}{l}\frac{\circ}{\grave{T}} \\
\frac{\grave{1}}{\bar{亠}}\end{array}$ & $\begin{array}{l}\frac{0}{2} \\
\frac{i}{\bar{\alpha}} \\
\text { ì }\end{array}$ & $\begin{array}{l}\frac{\bar{o}}{\bar{T}} \\
\frac{\bar{q}}{m}\end{array}$ & 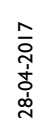 & 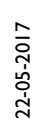 & $\begin{array}{l}\hat{\bar{a}} \\
\dot{\phi} \\
\stackrel{\dot{\omega}}{0}\end{array}$ & $\begin{array}{l}\hat{\bar{a}} \\
\dot{\leftrightarrow} \\
\stackrel{i}{N}\end{array}$ & $\begin{array}{l}\hat{\bar{c}} \\
\dot{\phi} \\
\dot{d} \\
\text { d. }\end{array}$ & 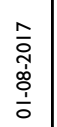 & $\begin{array}{l}\hat{\bar{c}} \\
\dot{\omega} \\
\grave{d} \\
\delta\end{array}$ & 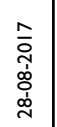 & $\begin{array}{l}\overline{\bar{a}} \\
\frac{\grave{T}}{\bar{亠}}\end{array}$ & 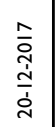 & 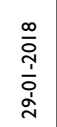 & 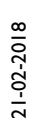 & 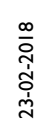 \\
\hline
\end{tabular}

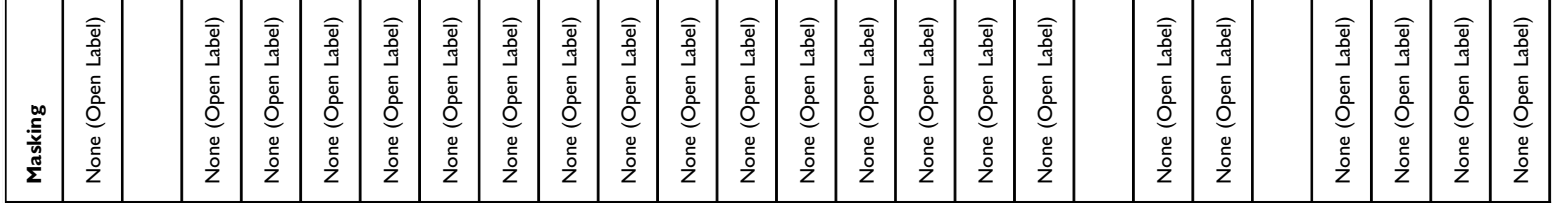

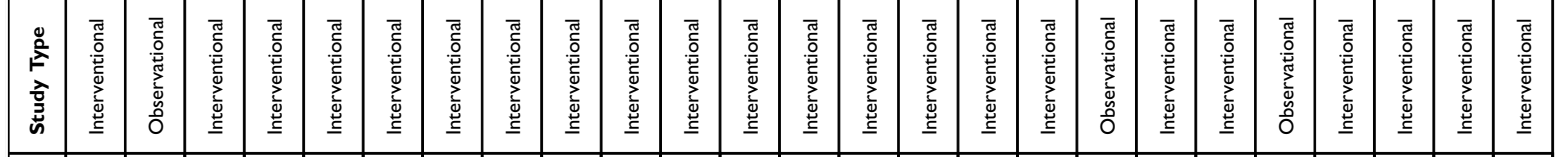

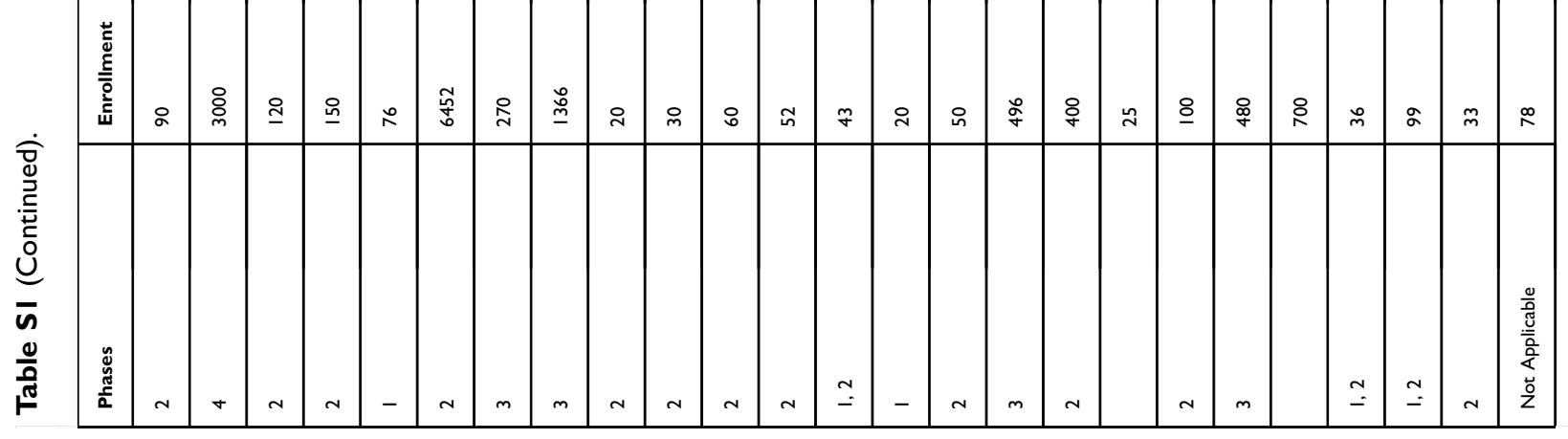




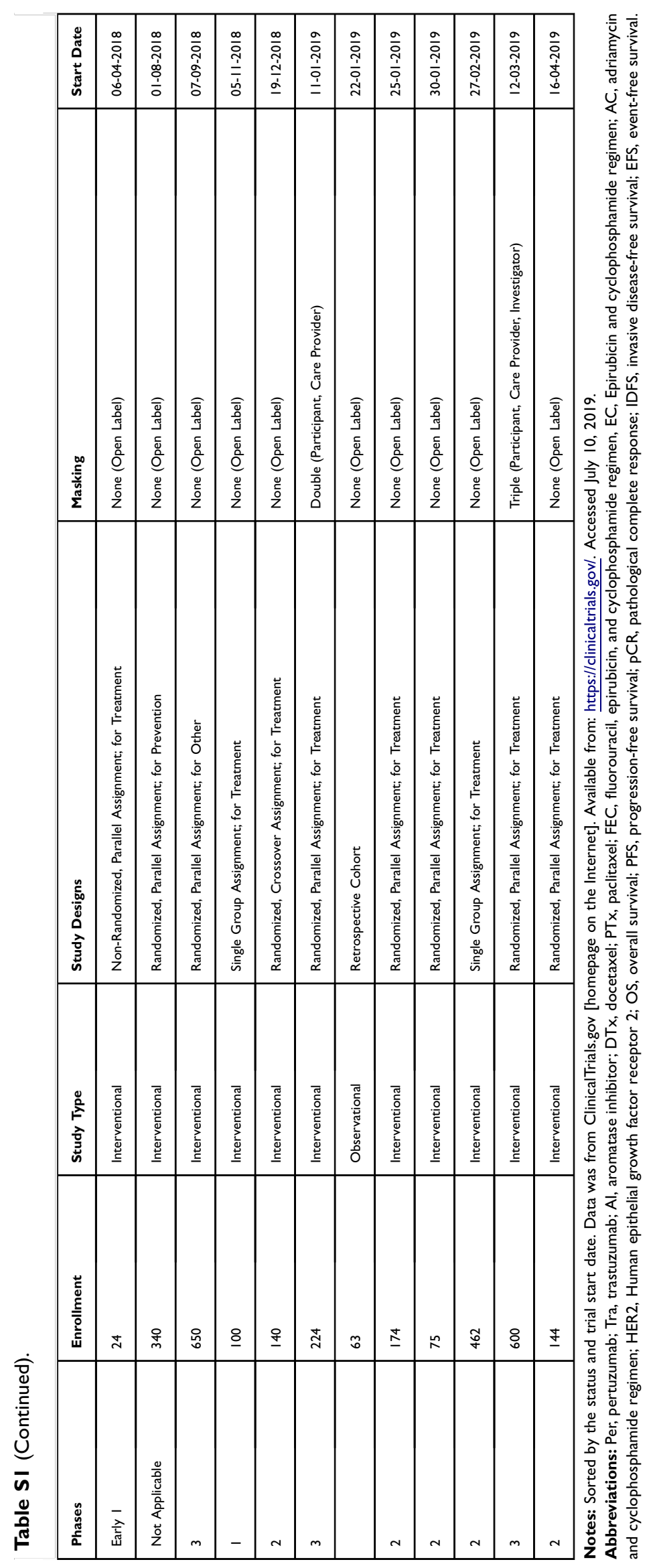




\section{Publish your work in this journal}

Core Evidence is an international, peer-reviewed open-access journal evaluating the evidence underlying the potential place in therapy of drugs throughout their development lifecycle from preclinical to post launch. The focus of each review is to evaluate the case for a new drug or class in outcome terms in specific indications and patient groups.

Submit your manuscript here: https://www.dovepress.com/core-evidence-journal
The manuscript management system is completely online and includes a very quick and fair peer-review system, which is all easy to use. Visit http://www.dovepress.com/testimonials.php to read real quotes from published authors. 